$\widehat{G}_{\text {http://dx.doi.org/10.3765/sp.8.9 }}^{\text {Semantics \& Pragmatics Volume 8, Article 9: 1-70, } 2015}$

\title{
Incremental quantification and the dynamics of pair-list phenomena*
}

\author{
Dylan Bumford \\ New York University
}

Submitted 2014-09-02 / First decision 2014-10-10 / Revision received 2014-10-29 / Accepted 2014-11-03 / Final version received 2014-11-17 / Published 2015-05-13

\begin{abstract}
Distributive universals are unique among natural language quantifiers in the following three ways: (i) matrix interrogatives that contain them accept pair-list answers; (ii) indefinites and disjunctions in their scope may assume arbitrary functional readings; and (iii) they permit sentence-internal interpretations of a wide range of comparative adjectives, like new and different. Because other quantifiers in the same environments do not give rise to these interpretations, the constructions provide a window into the semantic processes that underlie quantificational distributivity. In fact, both pair-list and internal readings have been independently argued to expose some of the compositional clockwork behind universal quantification, but the mechanisms they have been taken to reveal are entirely distinct. In contrast, I propose to explain pair-list phenomena, including a class of functional readings not previously recognized as such, and internal readings of comparative adjectives as two sides of the same coin; they are both side effects of incremental quantification. To make this precise, I analyze distributive universal quantifiers in terms of iterated, incremental update, in effect generalizing the sequential conjunction operator of standard dynamic semantics. I argue that this approach captures the tight empirical connection between pair-lists and internal adjectives, and at the same time provides a simpler and more robust account of the data than the specialized alternatives.
\end{abstract}

Keywords: universal quantification, pair-list readings, dynamic semantics, conjunction, functional readings, exceptional scope

* I would like to thank Chris Barker, Adrian Brasoveanu, Lucas Champollion, Simon Charlow, James Collins, Jeremy Kuhn, Salvador Mascarenhas, Prerna Nadathur, Philippe Schlenker, Anna Szabolcsi, two anonymous S\&P reviewers, the NYU Semantics Group, and audiences at the 19th Amsterdam Colloquium, Stanford Construction of Meaning Workshop, and UCSC S-Circle. This material is based upon work supported by the National Science Foundation Graduate Research Fellowship Program.

(C)2015 Dylan Bumford

This is an open-access article distributed under the terms of a Creative Commons Attribution License (http://creativecommons.org/licenses/by/3.o/). 
Dylan Bumford

\section{Introduction}

One way to answer the question in (1) is to specify for every student a particular novel that that student read, as in (1a). Such pair-list responses decompose what would appear to be a singular question about a family of individuals into a family of questions about singular individuals. The response is then a composite of answers to the individual sub-questions of the original interrogative.

(1) Which of Dickens's books has each student read?

a. Max has read Oliver Twist; Ken, Bleak House; and Oscar, David Copperfield.

[May 1985, Chapter 2, (18)]

Pair-list answers of this sort are decidedly less natural in response to questions with non-universal or non-distributive quantifiers (see e.g., Szabolcsi 1997). For instance, though the question in (2) could in principle be answered by specifying, for each of at least three dogs, a boy that it bit - or the question in (3) by specifying, for each host, some guest that he or she didn't want to invite - these responses are in fact infelicitous.

(2) Which boy did more than two dogs bite?

a. \#Fido bit $X$; Spot bit $Y$; King bit $Z$; ... [Szabolcsi 1997, (24)]

(3) Who did no one want to invite to the party?

a. \#Bill didn't want to invite Mary; Sam, Joe; and Nancy, Lucy.

[Engdahl 1985, (13)]

That the felicity of such answers depends on the type of quantifier in the question is important. As Szabolcsi (2010, p. 85) puts it, "What range of expressions actually participates in a given process is suggestive of exactly what that process consists in." So the connection between distributive universal quantifiers and pair-lists tells us something about the way distributive quantification works, in contrast with other types of quantification. At the very least, it suggests that universal DPs of the every/each variety interact with interrogatives in a way that other DPs do not.

But in what follows, I argue that pair-list phenomena are not limited to interrogatives. The same semantic mechanisms that permit pair-list answers to universal questions also explain the arbitrary functional interpretation of 
Incremental quantification

conditionals like (4) and the internal interpretation of sentences with comparative adjectives like (5). The relevant truth conditions are characterized by the formulas beneath the sentences, and will be discussed in detail in Section $2{ }^{1}$

1 A note on the notation used in this paper.

Judgments I mark naturally occurring examples with the ${ }^{\gamma}$ diacritic, ungrammatical examples with the traditional *, marginal examples with ?, and examples missing a relevant reading with \#. All judgments reported beneath cited example sentences are those of the cited authors.

Model Assumptions Models consist of the usual entities, relations, and functions. Modeltheoretic objects are displayed in sans-serif. When relevant, I assume entities in the model are mereologically ordered, where $x \oplus y$ represents the least upper bound of $x$ and $y$, and $\ll$ relates sums with their atomic parts.

Language and Metalanguage Mentions of English words are italicized in the running text. Lambda terms characterizing denotations are syncopated, so that $\lambda x_{0} \cdots x_{n} . M$ abbreviates $\lambda x_{0} \cdot \cdots \lambda x_{n} . M$. As usual, the bodies of such terms appeal freely to standard concepts and conventions from set theory and first-order logic. Perhaps most idiosyncratically: for any predicates $\mathrm{p}$ and $\mathrm{q}$ and set $\alpha$, (i) restricted variables of the form $x: p$ range over the subset of their type domain that satisfies $p$; (ii) $p \rightarrow q$ is the set of functions with domain $p$ and codomain $q ; p \hookrightarrow q$ the set of injections from $p$ into q; (iii) for any binary operator $\odot$ of type $\alpha \rightarrow \alpha \rightarrow \alpha$, the generalized unary operator $\odot$ of type $\{\alpha\} \rightarrow \alpha$ is defined recursively: $\odot\left\{a_{1}, \ldots, a_{n}\right\}=a_{1} \odot \odot\left\{a_{2}, \ldots, a_{n}\right\}$, where $\bigodot\{a\}=a$ for any singleton set $\{a\}$.

Type Conventions Unary types include entities $e$, truth values $t$, and discourse contexts $\sigma$ (modeled here as lists of entities). Constructed types take one of the following forms, where $\alpha$ and $\beta$ are any two types:

- $\alpha \rightarrow \beta$, the type of a function from $\alpha$ to $\beta$.

- $\{\alpha\}$, the type of a set of $\alpha$ objects.

- $\alpha \times \beta$, the type of an $\alpha$ object paired with a $\beta$ object, in that order.

Bracketing Conventions Function application is left associative; type descriptions are right associative. Parentheses are omitted, except where necessary for grouping. Periods separate variable-binding operators from the expressions they scope over, and have lower precedence than everything except for braces and slashes.

List Conventions If $s=\left[s_{n}, s_{n-1}, \ldots, s_{1}\right]$ is a list of objects, then $s_{k}$ picks out the $k$ th element from the end of $s$ (where $s_{1}$ is the last element on the list). Items are appended with the $\cdot$ operator: $s \cdot x=\left[s_{n}, s_{n-1}, \ldots, s_{1}, x\right]$. The empty list is represented by $\varepsilon$. 
(4) If each student makes progress in \{some, a (certain)\} area, nobody will flunk the exam.

$\exists f:$ student $\rightarrow$ area. $(\forall x$ : student .improve $(f x) x) \Rightarrow \neg \exists y$. flunk $y$

[Schlenker 2006, (23)]

(5) Every boy recited a different poem.

$\exists f:$ boy $\hookrightarrow$ poem. $\forall x$ : boy. recite $(f x) x \quad$ [Brasoveanu 2011, (3)]

Solomon (2011) suggests that these three classes of readings are connected, but does not explore that connection in any detail. Schlenker (2006) also draws attention to similarities between conditionals like (4) and pair-list questions, but only in passing. On the flip side, as far as I am aware, no analysis of the internal readings that sentences like (5) display so much as mentions pair-lists, though the two phenomena cast exactly the same compositional silhouette: the relevant interpretations only emerge when a singular indefinite appears in the scope of a distributive universal quantifier.

I propose that this is because only distributive universals trigger iterated dynamic conjunction over the alternatives in their scope. The basic idea is familiar: the universal claim in (6) reduces to the conjunction of propositions formed by mapping the property of reading a book over the set of students. What is unfamiliar is the idea that these conjuncts are evaluated in sequence, rather than in parallel. In dynamic frameworks, sentences typically denote updates over contexts. They move the current body of common information along from one state to another. Sentential conjunction composes the updates signaled by its conjuncts: the output from the first update becomes the input to the second. In this manner, earlier conjuncts add information to the common ground, which later conjuncts then access and refine. What emerges is an articulated mesh over the possible witnesses for the individual sentences. For instance, the composite update denoted by (6) sends an arbitrary input context $g$ to an output context that witnesses each student's reading some particular book. That is, any potential state of affairs that is compatible with the information provided by (6) will effectively pair every student with a book that he or she read.

(6) Every student read a book.

$\llbracket$ John read a book $\rrbracket$; Mary read a book $\rrbracket ; \llbracket$ Fred read a book $\rrbracket$ 
Incremental quantification

Interestingly, sentential conjunction has the same refining effect on alternatives in a variety of static semantic frameworks, including Alternative Semantics (e.g., Kratzer \& Shimoyama 2002), Inquisitive Semantics (e.g., Roelofsen 2013), Truthmaker Semantics (e.g., Fine 2013), and more generally, any semantics that models indefiniteness as semantic indeterminacy (e.g., with sets of propositions). However, dynamic evaluation in particular also accounts for the possibility of quantifier-internal anaphora, in which an adjective like different compares objects across the alternatives that $e v$ ery quantifies over. Because later conjuncts are evaluated in the anaphoric context of earlier conjuncts, the semantic commitments induced by early information can constrain the possible interpretations of subsequent information. Impressionistically, the algorithm that interprets ( 7 ) first selects a book that witnesses John's readership, and only then - with that book in mind, so to speak - searches for a different book to witness Mary's readership, and so on. ${ }^{2}$

(7) Every student read a different book.

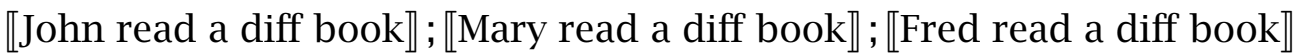

In addition, the incremental evaluation strategy novelly predicts the availability of asymmetric internal readings, like that of (8). These sorts of sentences exhibit the same distributional restrictions as symmetric internal readings (Beck 2000, Brasoveanu 2011), pair-list conditionals, and pair-list answers, which provides further evidence that they all flow from the same semantic spring.

(8) Every year Mary wrote a more interesting book.

【In 2011 Mary wrote a more interesting book》;

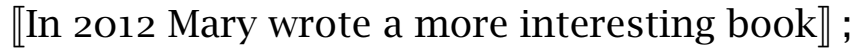

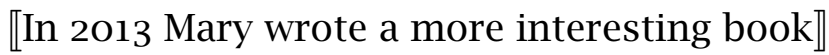

The rest of the paper is organized as follows: Section 2 reviews the evidence accumulated in different literatures that both pair-list readings and internal readings depend in precisely the same way on distributive universal quantification. Section 3 describes the incremental update strategy in detail, and Section 4 implements the analysis in the dynamic setting of Charlow

2 See also Brasoveanu 2011 for a very different dynamic analysis of quantifier-internal anaphora, discussed in Section 6. 
2014. Charlow's grammar provides a nice interface between dynamic binding and exceptional scope-taking. This lays the compositional groundwork for the essentially incremental, dynamic effects of (7) and (8), as well as the essentially scopal pair-list effects of (1) and (4). Then in Section 6, I compare the incremental approach to other proposals in the intellectual vicinity.

\section{Core data}

Internal interpretations of comparative adjectives on the one hand, and pairlist interpretations of questions and embedded clauses on the other, are only possible when a distributive universal quantifier (e.g., every student) outscopes an indefinite (e.g., a book). In this section, I review the basic evidence in support of these generalizations.

\subsection{Internal adjectives}

\subsubsection{Internal readings of different}

Comparative adjectives like same and different are put to a wide variety of semantic uses, which have been cataloged and characterized by Stump (1982), Dowty (1985), Heim (1985), Carlson (1987), and Keenan (1987), among others. Some of these uses are demonstrated in (9). In particular, Carlson (1987) distinguishes external uses, in which at least one of the things that the adjective compares is implicit or provided by the surrounding discourse, from overt, reciprocal, and internal uses, in which the elements to be compared are all present in the sentence, one way or another.

\section{(9) Varieties of different}

a. Smith went to a different city on his vacation this year. external

b. You answered a different question than the one I asked. overt

c. John saw several different animals at the zoo. reciprocal

d. Bob and Alice attend different classes. internal

e. Each man is from a different town. distributive internal [cf. Carlson 1987, (1-4)]

The latter three are further distinguished by the grammatical location of the comparates. Overt comparisons exploit a full than or as clause to 
specify the target of comparison. Reciprocal uses compare a handful of objects to themselves. Internal uses consider the individuals indicated by some expression independent of the different phrase, and compare those individuals with respect to a property that includes the different phrase. For example, on its internal reading, (9d) says that Bob and Alice differ with respect to the classes that they attend.

Dowty (1985) and later Beck (2000) and Brasoveanu (2011) further subdivide the internal readings into distributive (9e) and non-distributive (9d) uses. Beck argues that non-distributive internal readings are special cases of the reciprocal readings exemplified in (9c). On her view, the different DP in (9c) requires that some unspecified subsets of the animals at the zoo be distinct. Similarly, the different DP in (9d) requires that some subsets of classes be distinct, but in this case the relevant subsets are made salient by the sentential context; namely, the classes attended by Bob and the classes attended by Alice.

Beck's reasons for drawing the line between plural different (which includes reciprocal and internal uses) and singular different are empirical, motivated by differential scopal behavior and congruences with overt reciprocals (e.g., each other), as analyzed in Schwarzschild 1996 and Heim, Lasnik \& May 1991. For instance, singular DPs resist internal readings in a range of contexts that support internal readings for plural DPs. (10) gives representative contrasts for singular/plural DPs in the scope of conjunctions and definite plurals. Dowty (1985), Carlson (1987), and Moltmann (1992) all attribute this contrast to a requirement that internal different DPs agree with their "antecedents" in morphological number. Dotlačil (2010) and Brasoveanu (2011), however, observe that DPs headed by no fail to license internal readings, even when they are morphologically singular, as in (11).

(10) a. \{John and Mary, The boys $\}$ found different solutions.

b. \#\{John and Mary, The boys $\}$ found a different solution.

[Moltmann 1992, (88)]

(11) \#In the class, no boy read a different book.

[cf. Dotlačil 2010, Chapter 5, (112)]

Instead, after an extensive survey of universal and (in)definite DPs in the Corpus of Contemporary American English (COCA), Brasoveanu concludes that as far as universal DPs are concerned, only the genuinely distributive ones like every, each, and whenever support internal readings of 
singular different. This excludes, for instance, definite plurals, conjunctions, for-adverbials, and aspectual modals like continuously. Beghelli (1997) and Beghelli \& Stowell (1997) come to an even stronger conclusion, echoed later by Solomon (2011): no DPs of any sort license internal readings, except for the distributive universals. See, for example, the contrasts in (12).

(12) a. $\{$ Every, each\} boy read a different book.

[Beghelli \& Stowell 1997, (20a, b)]

b. \#\{All, both $\}$ of the boys read a different book. [Brasoveanu 2011, (18)]

c. \#(More than) Five boys read a different book.

[Beghelli 1997, (23C, e)]

d. \#Many (of the) boys read a different book. [Solomon 2011, (96)]

\subsubsection{Internal readings of morphological comparatives}

As might be expected, the syntactic profile of different is very close to that of run-of-the-mill morphologically comparative adjectives like taller, and the two classes of adjectives enjoy a similar variety of uses (Heim 1985, Beck 2000). In particular, morphological comparatives have external anaphoric uses as in (13a), uses with overt comparison clauses (13b), and singular internal uses within the scope of distributive quantifiers (13c).

(13) a. Smith went to a \{bigger, different city on his vacation this year. [cf. (1)]

b. Ornette Coleman played a \{louder, different $\}$ tune than Don Cherry. [Heim 1985, (28)]

c. She gave a $\{$ better, different $\}$ talk every time. [Beck 200o, (90)]
[

In fact, both Beck (2000) and Brasoveanu (2011) maintain that, just as in the case of singular different, internal interpretations of singular DPs containing morphological comparatives are only possible in the scope of distributive universals. Because this observation has received less attention than the analogous generalization concerning different, here are a few more examples from COCA, reported in Brasoveanu 2011. (I use ${ }^{\gamma}$ superscripts here 
Incremental quantification

and throughout to mark naturally occurring examples; see footnote 1 for a complete description of notational conventions.)

(14) a. ${ }^{\gamma}$ Every second I am becoming more outnumbered.

[Brasoveanu 2011, (201)]

b. ${ }^{\gamma}$ It's more dangerous every day that they appear in court.

[Brasoveanu 2011, (202)]

c. ${ }^{\gamma}$ Each generation inhabits a progressively more Orwellian world. [Brasoveanu 2011, (207)]

d. \#\{Those, Several, Most, $\emptyset\}$ generations inhabit a progressively more Orwellian world.

\subsubsection{Internal readings of other comparative adjectives}

There are still other comparative adjectives with internal uses in explicitly distributive contexts, including new, other, and fresh. Attested examples are given in (15). ${ }^{3}$

(15) a. ${ }^{\gamma}$ Every day this calendar reveals a new Polaroid photo, each with its own little story.

b. ${ }^{\gamma}$ Every day, we publish a new short story of 1000 words or fewer that can be read during your lunch hour, on transit, or even over breakfast.

c. ${ }^{\gamma}$ Cute cottages, each one another color!

d. ${ }^{\gamma}$ Marriage is like a coffin, and each kid is another nail.

e. ${ }^{\gamma}$ HobNob Communications promises each client a fresh media relations campaign, no stale messages or repackaged programs.

3 Brasoveanu (2011) actually presents other as an example of a comparative adjective that refuses quantifier-internal interpretations, on the basis of (i), which he judges to be unambiguous and true iff every boy recited a poem other than "The Raven". I leave it as an open question how to predict when other can and cannot be read quantifier-internally.

(i) Mary recited "The Raven". Then every boy recited another poem.

[Brasoveanu 2011, (72b)] 
f. ${ }^{\gamma}$ Subsequently transfer $1 \mathrm{ml}$ of previous virus dilution to next dilution by using at each step a fresh pipette, to achieve serial tenfold dilutions.

All of these internal readings disappear in non-distributive contexts, even when the number of the relevant DP shifts accordingly. (16) and (17) provide representative contrasts. The sentences in (17) all require sentence-external anaphors; the sentences in (16) do not.

(16) a. ${ }^{\gamma}$ Motorola announced device after device, each with a more complicated name. The DroID RAzR HR, DroId RAzR MAzz HR [...]

b. ${ }^{\gamma}$ This is how I plan to get through the next year. Every day, I will post another thing to keep me looking forward and keep from getting mired down in the bad times.

c. ${ }^{\gamma}$ The robot ants go marching one by one, each laying down a new layer of light.

(17) a. \#Motorola announced device after device, all with \{a more complicated name, more complicated names\}.

b. \#Throughout the next year, I will regularly post \{another thing, other things\} to keep me looking forward.

c. \# The robot ants go marching one by one, laying down \{a new layer of light, new layers of light

The empirical moral of this subsection, then, is that singular comparative adjectives of all stripes permit internal interpretations when - and only when - they occur in the scope of a distributive universal quantifier. Despite a tradition of semantic research into internal readings stretching back at least 30 years, the incremental analysis sketched above and spelled out in Section 4 is the first compositional theory to date that accounts for both symmetric and asymmetric adjectives, as well as their connection to universals.

\subsection{Pair-list questions}

The sentence in (18) can be answered by any of (18a-c), known respectively as its individual answer, its pair-list answer, and its functional answer. The first two are intuitively distinguished by the relative scopes of the universal 
Incremental quantification

and $w h$-DP. The third has received much attention, as it seems to require a nonstandard "functional" interpretation of the wh-word and the constituent answer (e.g., Engdahl 1980, Groenendijk \& Stokhof 1984, Chierchia 1993, Jacobson 1999, Sharvit 1999, Winter 2004, Barker 2009).

(18) Which woman does every man love?
a. Mary.
individual answer
b. Alex loves Ann, Bill loves Bee, Carl loves Cat.
pair-list answer
c. His mother.
functional answer

[Groenendijk \& Stokhof 1984, Chapter 3, (1)]

Functional answers like (18c) are felicitous in a wider range of contexts than pair-list answers like (18b). In particular, a question like (18) may be answered functionally no matter what kind of DP appears in place of every man. The sentences in (19), adapted from Krifka 2001, provide some examples.

(19) Which dish did \{all the, most, several, few, no\} boy(s) make?

a. \{His, Their $\}$ favorite dish. [cf. Krifka 2001, (5)]

In contrast, pair-list answers to quantificational questions are usually quite unnatural. Groenendijk \& Stokhof (1984, Chapter 3) initially claimed that arbitrary pair-list answers were only available to questions ranging over "universal terms" (essentially names, conjunctions, definite plurals, and distributive quantifiers). Shortly thereafter (Groenendijk \& Stokhof 1984, Chapter 4), the list was extended to include bare numerals, though with some flexibility in the domain of the pair-list answer. Chierchia 1992 and 1993 follow the same empirical trajectory.

However, Krifka (1992) and Srivastav (1992) independently argue that apparent pair-list answers to questions ranging over definite plurals and conjunctions, as in (20), are actually very specific cumulative answers. They become infelicitous when the wh-DP is unambiguously singular, as in (21), because singular DPs do not participate in cumulative readings.

Along similar lines, Gawron \& Kehler (2003) argue that conjoined constituents do not support genuine pair-list interpretations either. So while (22b) provides an ostensible pair-list answer to the question in (22), here too, the answer becomes infelicitous when the wh-word is unambiguously singular (23). They propose instead that answers like (22b) are in fact instances of respectively-conjunctions, similar in many ways to cumulative readings. 
Dylan Bumford

(20) What did the boys rent last night?

a. (The boys rented) $Z$.

b. Al rented $A$, Bill rented $B$, and Carl rented $C$. [Krifka 1992, (3)]

(21) Which movie did the boys rent last night?

a. (The boys rented) $Z$.

b. \#Al rented $A$, Bill rented $B$, and Carl rented $C$. [Krifka 1992, (8b)]

(22) Where did Mary vacation and Bill decide to live?

a. Rome.

b. Mary vacationed in Paris and Bill decided to live in Toronto.

[Gawron \& Kehler 2003, (1)]

(23) In what city did Mary vacation and Bill decide to live?

a. Rome.

b. \#Mary vacationed in Paris and Bill decided to live in Toronto.

[Gawron \& Kehler 2003, (6)]

In addition, Szabolcsi (1997) questions the acceptability of pair-list answers to questions with bare numerals, and argues that to the extent that they are coherent, they too are examples of over-informative cumulative responses. The view is corroborated by Krifka (2001), who observes that speech acts in general refuse to scope beneath disjunctions of any form. As a special case of this resistance, interrogatives containing bare numeral indefinites are strongly preferred with $w h>\exists$ scope, which rules out pair-list answers. More generally, according to Krifka, pair-list interpretations are at best highly marginal for any questions containing DPs with multiple witness sets.

Taken together, these observations add up to a simple generalization: the only questions that may be felicitously answered by specifying a list of witnessing pairs are those that contain a distributive universal quantifier.

\subsection{Schlenker readings}

Declarative sentences with indefinite DPs recruit the same variety of interpretations as the interrogatives sampled above. For instance, (24), like the analogous question in (18), is three-ways ambiguous between a surface scope, 
Incremental quantification

inverse scope, and functional interpretation of the indefinite. The respective readings are brought out by the three continuations in $(24 \mathrm{a}-\mathrm{c})$.

(24) Every man loves a woman. Namely, ...

a. Mary.

individual continuation

b. Alex loves Ann, Bill loves Bee, Carl loves Cat. pair-list continuation

c. His mother.

functional continuation [Groenendijk \& Stokhof 1984, Chapter 3, (33)]

The first two continuations pick out the two possible scopal arrangements of the standard generalized quantifiers denoted by the two DPs. The third, like the first, interprets the indefinite DP with broader scope than the universal. But unlike the first, it interprets the DP as an existential quantifier over functions; specifically, functions into the set of women.

With declaratives as with interrogatives, it is important to distinguish these functional interpretations from pair-list interpretations like (24b). Groenendijk \& Stokhof (1984) observe that functional interpretations are available even to sentences in which a universal is separated from its indefinite by a scope island, though pair-list readings are not.

(25) There is a woman whom every man loves. Namely, ...
a. Mary.
b. \#Alex loves Ann, Bill loves Bee, Carl loves Cat.
c. His mother.
[Groenendijk \& Stokhof 1984, (36)]

In the same vein, von Stechow (1990), Jacobson (1994, 1999), Sharvit (1999), and Winter (2004), among others, discuss copular sentences like the one in (26), in which a complex DP is explicitly identified with a particular $e \rightarrow e$ function. Here, (26) is taken to mean that the only (relevant/natural/salient) function that assigns every Englishman to the woman he most reveres is the function that assigns each man to his mother. Again, because the universal quantifier is trapped within a relative clause, it cannot legitimately outscope the definite article, and as a result the sentence has no pair-list interpretation.

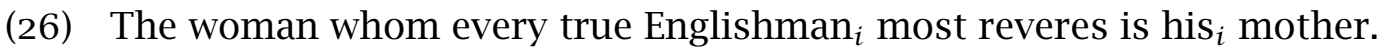
$(\iota f . \forall x$ : englishman . revere $(f x) x)=(\lambda x: \operatorname{man} . \operatorname{mom} x)$

[Geach 1964, (2)] 
Hintikka (1986) likewise exploits quantification over $e \rightarrow e$ functions to untangle the branching scopal interactions of sentences with a certain indefinites. The sentence in (27), for example, can be true in virtue of my knowing that (26) is true. More precisely, according to Hintikka, (27) does not merely indicate that I know that it's possible to pair Englishmen with women they love, but rather that for some such pairing, I know that every Englishman adores the woman he is paired with. The latter state of affairs, formally paraphrased below (27), is not derivable under any linear scoping of the three logical operators in the sentence (know, every, a), and so a fortiori cannot be a special case of the pair-list configuration (see Hintikka 1986 and Schlenker 2006 for details).

(27) I know that every true Englishman adores a certain woman.

$$
\exists f . \square_{\mathrm{i}}(\forall x \text { : englishman. adores }(f x) x) \quad \text { [Hintikka 1986, (19)] }
$$

What's more, just as the questions in (19) may all be answered by naming an appropriate function, these functional indefinites are in general insensitive to the sort of quantifier that binds into them. Quantificational analogs of (26) and (27) are given in (28) and (29).

(28) The woman whom \{all the, most, several, few, no\} true Englishmen adore is their mother.

(29) I know that \{all the, most, several, few, no\} true Englishmen adore a certain woman; namely, their mother.

However, it is more difficult to characterize the precise conditions under which pair-list readings are licensed in declarative contexts. In fact, so far in this section, I have just identified the pair-list reading with the $\mathcal{Q}>\exists$ scopal configuration, which in simple cases like (24) is truth-conditionally equivalent to the functional interpretation anyway. The continuation technique from (24) helps to tease the two interpretations apart, but it cannot distinguish quantifiers that license genuine pair-list readings from those that don't, because any quantified statement may be elaborated upon by spelling out some or all of the facts that verify it, as in (30a).

(30) Most men love a certain woman....

a. Alex loves Ann, Bill loves Bee, and Carl loves Cat.

b. Their mother. 
Incremental quantification

Nevertheless, there is an empirical distributional difference between the pair-lists generated by distributive universals like every and those that are compatible with other quantifiers like most. The key is in the Hintikka sentence, (27). The value of this example is that it manages to wedge a third logical operation in between the quantifier over functional witnesses and the universal that binds into it. As mentioned above, this truth-conditionally distinguishes the functional reading from the unadorned surface scope reading, and as a result provides solid evidence for the availability of independent functional interpretations of indefinites.

Crucially, Schlenker $(1998,2006)$ offers evidence that genuine, arbitrary pair-list witnesses for embedded universal clauses exhibit the same scope flexibility as these his mother-style functional indefinites. He observes that the sentence in (31) can describe a situation in which all of the students have a particular weakness, and in order for the class to achieve success on the exam, each student must improve in the particular area that troubles him or her.

(31) If each student makes progress in \{some, a (certain)\} area, nobody will flunk the exam. [repeated from (5)]

As before, this meaning - that total passage is guaranteed by a particular distribution of improvements - cannot be derived from any linear scoping of the $\forall, \exists$, and $\Rightarrow$ operators. On the surface then, this would appear to be just another case of the Hintikka-style sentence exemplified in (27), in which the indefinite triggers an exceptionally-scoped existential quantifier over functions from students to the areas that they need to improve in.

The problem is that this is decidedly not the sort of function Hintikka had in mind. He in fact explicitly conjectures that "[w]hen there is no simple familiar function in the offing as a value of a function quantifier like $(\exists f)$ in $[(27)]$, the reading analogous to [(27)] is likely to be unnatural.” Groenendijk \& Stokhof (1984) come to much the same conclusion:

[I]f one asks for the specification of a function (with a question on its functional reading), or asserts the existence of a function and gives a specification of it, one obviously is not satisfied with any old specification of any old weird functional relationship between individuals. [...] It would seem that the functions that are allowed, must be either conventional in some sense (such as the mother-function, the wife-function, etc.) and thus in some 
Dylan Bumford

sense computable, or they must be made computable by the context.

Chierchia (1992, 2001), Dayal (1996), Kratzer (1998), and Sharvit (1996, 1997) all express similar views, and it is implicit or taken for granted in Engdahl 1986 and much of the work on functional relative clauses (e.g., Jacobson 1994, 1999). Schwarz (2001) considers at length the rather dramatic potential for unconstrained functional quantification to overgenerate. For example, if functional variables are free to range over "any old weird functional relationship", then the sentence in (32) should have a very weak reading according to which it is true as long as no student read every book I recommended (see Schwarz for details). Of course it has no such reading.

(32) No student read a book I had recommended.

$\# \exists f:$ student $\rightarrow$ book. $\neg \exists y:$ student . read $(f y) y \quad$ [Schwarz 2001, (18)]

Spector (2004) contends that these sorts of arbitrary, wide-scope pair-list interpretations are similarly absent for conditionals containing conjunctions, plurals of all sorts, and proportional quantifiers. Some of Spector's examples and judgments are reported in (33). Solomon (2011) reports similar facts for negative quantifiers like that in (34), and reviewing the evidence, concludes that only distributive universals interact with plain indefinites in such a way as to reliably produce non-conventionalized pair-list readings.

(33) \#If \{many students, all the students, Alex and Bill $\}$ study a certain topic, the exam will be a success. [Spector 2004, (9-11)]

(34) \#If no student makes progress in a certain area, everybody will flunk.

[Solomon 2011, (22)]

To bring out the judgments a little more clearly, imagine a simple slot machine with three reels and a handful of symbols (it doesn't matter how many). Given standard assumptions about slot machines, the sentence in (35a) seems true on its pair-list reading, even if nobody knows which combination(s) of symbols will actually win. That is, the sentence is true not because of any particularly salient or natural function mapping reels to symbols, but because that's just how slot machines work: there are some arrangements of symbols that earn you money, and a lot that don't. 
Incremental quantification

At the same time, this sort of interpretation is entirely unavailable to (35b). It cannot be understood as conveying that your loss is guaranteed when none of the reels land on the symbol that some particular function assigns them. Importantly, this is the case even though any natural or salient function that might witness the truth of (35a) might just as easily witness the truth of (35b). Put another way, if the pair-list reading of (35a) were just a special case of a functional reading - one in which the winning pattern of symbols were somehow made salient by context - then it's hard to see why (35b) would so strongly resist an analogous reading, given that the very same function in the very same context would verify them both.

(35) a. If every reel lands on a certain symbol, you'll win the prize. $\exists f: \mathbb{R} \rightarrow S .(\forall x: \mathbb{R}$. land $(f x) x) \Rightarrow$ win p you

b. If no reel lands on a certain symbol, you'll lose. $\# \exists f: \mathbb{R} \rightarrow S .(\neg \exists x: \mathbb{R}$. land $(f x) x) \Rightarrow$ lose you

\subsection{Summing up and pushing forward}

Here's where the data stand:

i. An internal reading of a comparative adjective in a singular DP is only possible in the scope of a distributive universal quantifier (Beghelli 1997, Brasoveanu 2011). ${ }^{4}$

ii. A pair-list answer to a singular wh-question is only felicitous when the $w h$-word is interpreted in the scope of a distributive universal quantifier (Szabolcsi 1997, Krifka 2001).

4 I am grateful to an anonymous reviewer who points out that recent experimental work reported in Brasoveanu \& Dotlačil 2012 suggests that the licensing of internal adjectives may not be categorically restricted to the small class of lexicalized distributors that Beghelli and Brasoveanu identify. Brasoveanu \& Dotlačil's (2012) acceptability surveys, for instance, indicate that singular different is tolerated to some extent in the scope of definite plurals, especially those headed by all, though to a significantly lower degree than in the scope of each. This cline follows a general trend established by Dotlačil (2010): lexicalized distributors induce covariation in indefinites they outscope more readily than all-headed DPs, which in turn induce covariation more readily than bare definite plurals. As the reviewer observes, this research gives reason to suspect that the proper empirical generalizations concerning pair-list questions and embedded clauses in (ii) and (iii) might in reality be graded along the same distributive slope: each $>$ all $>$ the. This is an exciting conjecture, confirmation of which will have to await further investigation. 
iii. An embedded clause containing a singular indefinite DP is only sensitive to an exceptionally-scoping pair-list association when the indefinite is interpreted in the scope of a distributive universal quantifier (Spector 2004, Schlenker 2006, Solomon 2011).

I am not the first to point out any of (i), (ii), or (iii), and all of these facts have received analyses in their respective literatures. Brasoveanu (2011), for instance, treats internal readings of different in a specialized dynamic framework that only every and different can fully exploit. Groenendijk \& Stokhof $(1984,1989)$ derive pair-list questions from a special sort of QR mechanism that interprets quantifiers in questions as domain restrictors on relations, an operation which is incompatible with downward-entailing and nonmonotonic DPs. Schlenker (2006) accounts for the exceptional reading of (31) by appealing to existential closure over Skolemized choice functions.

Unfortunately, none of these analyses extends in a natural way to explain the nearby generalizations. This could be acceptable if it turned out that distributive universals have multiple independent properties, some of which interact with comparative adjectives, and others of which give rise to pair-list readings in various contexts. But I'll argue in the next section that all three of these phenomena emerge as side-effects from one small, simple change in the way we typically think of universal quantification. And in Section 6, I'll argue that this way of deriving the relevant readings is actually more adequate for each of the phenomena independently than the leading alternatives.

\section{Iterated conjunction generates pair-lists}

\subsection{Conjunction and disjunction in alternative-oriented semantics}

One way or another, many theories that trade in nondeterministic meanings have arrived at (36) as the semantics of disjunction. For instance, (Groenendijk \& Stokhof 1991, Definition 33) propose as a denotation for $A \vee B$ the relation over contexts containing every input-output pair in $A$ and every input-output pair in $B$. In Alternative Semantics and Inquisitive Semantics, the disjunction of sentences $A$ and $B$ is the set of alternatives/possibilities containing all of the propositions in $A$ and all of the propositions in $B$ (Alonso-Ovalle 20o9, (10), Roelofsen 2013, Definition 7). Recent versions of Truthmaker Semantics identify the set of verifiers for $A \vee B$ with the set containing everything that verifies $A$ and everything that verifies $B$ (Fine 2012, p. $234\left(\right.$ iii) ${ }^{+}$). And so on. 
Incremental quantification

$$
\llbracket \phi \vee \psi \rrbracket=\llbracket \phi \rrbracket \cup \llbracket \psi \rrbracket
$$

The subset of those theories that also worry about conjunction typically define it as the pointwise composition, fusion, or intersection of the alternatives in the two conjuncts. ${ }^{5,6}$

(37) a. $\llbracket \phi \wedge \psi \rrbracket=\{\langle g, h\rangle \mid \exists k .\langle g, k\rangle \in \llbracket \phi \rrbracket \wedge\langle k, h\rangle \in \llbracket \psi \rrbracket\}$

[Groenendijk \& Stokhof 1991, Definition 2]

b. $\llbracket \phi \wedge \psi \rrbracket=\left\{s \oplus s^{\prime} \mid s \in \llbracket \phi \rrbracket, s^{\prime} \in \llbracket \psi \rrbracket\right\} \quad$ [Fine 2012, p. 234 (ii) $\left.^{+}\right]$

c. $\llbracket \phi \wedge \psi \rrbracket=\{p \cap q \mid p \in \llbracket \phi \rrbracket, q \in \llbracket \psi \rrbracket\}$

[Ciardelli 2009, Definition 6.2.1]

What's important to note for present purposes is that in all of these frameworks, the arguments to a conjunction are finely articulated semantic objects. On the dynamic picture, they are inputs with potentially many outputs. On the inquisitive picture, they are sets containing potentially many alternatives. In either case, conjunction is designed to thread the possible updates/alternatives from the first conjunct individually into the possible updates/alternatives in the second conjunct, and then collect the results into a composite update or refined set of alternatives.

Of course, indefinites exhibit the same dynamic and alternative-generating behavior as disjunctions, and are treated accordingly in dynamic and inquisitive programs. That is, one way or another, existential quantification in all of these semantic guises comes out as equivalent to generalized disjunction, along the lines of (38). An indefinite first builds a set of potentially nondeterministic propositions by substituting into its nuclear scope all of the individuals determined by its restrictor (in this case, the entire domain), and then takes the grand union of the propositions, exactly as if iteratively disjoining them.

5 For Fine, sentences denote the sets of their verifiers, which are to a first approximation the facts or situations that make them true. As with other model-theoretic entities, the $\oplus$ operator here returns the mereological sum of its arguments, in this case the state comprised of $s$ and $s^{\prime}$ and nothing else.

6 Different versions of Inquisitive Semantics require slight variations on the theme in (37c). Earlier incarnations (e.g., Groenendijk \& Roelofsen 2009, Ciardelli \& Roelofsen 2011) restrict the possibilities of $\phi \wedge \psi$ to just the maximal intersections of $\phi$ and $\psi$ possibilities. Later variants (e.g., Roelofsen 2013) actually define $\llbracket \phi \wedge \psi \rrbracket$ as the direct intersection $\llbracket \phi \rrbracket \cap \llbracket \psi \rrbracket$, but because propositions in these variants are closed under possibility-subsets, this is equivalent to the pointwise intersection given in (37c) above. 
This guarantees that indefinites too will ramify the semantic context, and the indeterminacy they generate will trickle through conjunction. Take (39), for example. The first sentence denotes a set of updates/alternatives, each of which pairs John with a particular dog (that he owns). Likewise with the second, mutatis mutandis. Conjoining the two gives a doubly reticulated set of alternatives, each of which pairs John and Fred with particular dogs (that they own).

(39) a. \John has a $\operatorname{dog} \rrbracket \approx\{$ has $x \mathrm{j} \mid x \in \operatorname{dog}\}$

b. $\llbracket$ Fred has a $\operatorname{dog} \rrbracket \approx\{$ has $y \mathrm{f} \mid y \in \operatorname{dog}\}$

c. 【John has a dog; Fred has a $\operatorname{dog} \rrbracket \approx\{$ has $x \mathrm{j} \wedge$ has $y \mathrm{f} \mid x, y \in \operatorname{dog}\}$

In contrast, in standard dynamic fragments, universal quantification is not a generalization of dynamic conjunction (e.g., Groenendijk \& Stokhof 1991, 1990, Muskens 1996, de Groote 2006, Brasoveanu 2008, Charlow 2014). The example in (40) from Groenendijk \& Stokhof is representative. The universal simply tests that the propositions formed by substituting individuals from its restrictor into its scope all lead to successful updates. And then it very deliberately throws those updates away (more on this issue in Section 4.2.3). ${ }^{7}$

$$
\llbracket \forall x . \phi \rrbracket=\{\langle g, g\rangle \mid \forall k . k[x] g \Rightarrow \exists j .\langle k, j\rangle \in \llbracket \phi \rrbracket\}
$$

[Groenendijk \& Stokhof 1991, Definition 2]

But there's certainly nothing incoherent about generalized dynamic conjunction. In fact, Groenendijk \& Stokhof (1991) consider a related notion under the rubric of Program Repetition, defined in (41). Here, the iterative closure of a formula $\pi$ denotes any update that is itself composed of (possibly many) incremental updates satisfying $\pi$. For instance, the nondeterministic update $\llbracket[\text { John read a book }]^{*} \rrbracket$ is satisfied by any composition of updates that each witness John's reading some particular book. Groenendijk \& Stokhof discard the notion as irrelevant to natural language, and it seems to have never resurfaced, at least not in connection with natural language quantifiers.

7 Alternative Semantic treatments of universal quantification are harder to come by, but recent first-order variants of Inquisitive Semantics actually do define universal quantifiers in terms of generalized conjunction (Ciardelli 2009, Roelofsen 2013). However, the analysis of internal adjectives in Section 4 requires some theory of scope-shift and cross-sentential anaphora, neither of which have been worked out for the inquisitive paradigm. 
Incremental quantification

(41) $\llbracket \pi^{*} \rrbracket=\left\{\langle g, h\rangle \mid \exists \vec{j} \cdot \begin{array}{c}j_{0}=g, j_{n}=h, \\ \forall i \leq n \cdot\left\langle j_{i-1}, j_{i}\right\rangle \in \llbracket \pi \rrbracket\end{array}\right\}$

[Groenendijk \& Stokhof 1991, Definition 2]

Yet, with just a little fiddling, this sort of incremental quantification obviously could model the semantics of natural language universals, and in what follows, I'll argue that in fact it provides a better model for the semantics of universals than standard definitions. The idea is simple: a sentence like (6), repeated in (42a), denotes the composition of individual updates sketched in (42b), where John, Mary, and Fred are the students.

(42) a. Every student read a book.

[repeated from (6)]

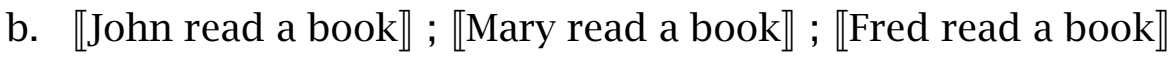

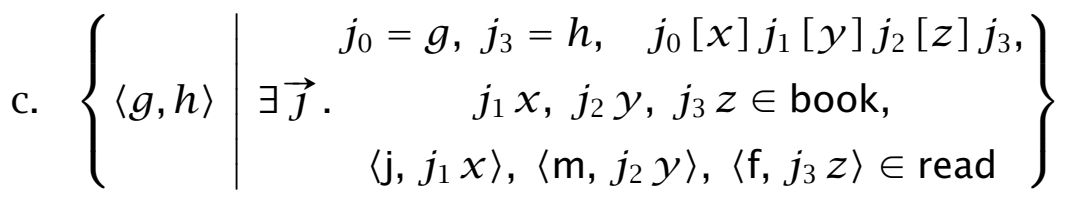

Any update satisfying the composition in (42b) will first pair John with a particular book, then Mary, then Fred. As before, the indeterminacy introduced by the indefinite will propagate through the computation, forking the possibilities at every conjunct. The ultimate composite update shown in (42c) relates any input context $g$ to any output context $h$ which is just like $g$, except that it maps the variables $x, y$, and $z$ to books that John, Mary, and Fred read, respectively. In other words, the output contexts all pair John, Mary, and Fred with particular books, and the entire sentence is true just in case at least one such pairing respects the read relation.

\subsection{Incremental quantification}

Those pairings, of course, are exactly the "pair-lists" that end up as answers to universal-embedding questions like (18), and with exceptional scope in Schlenker sentences like (31). In Section 4, I will say what it means exactly for a pairing to take scope. But first note that because the "lists" here are assembled incrementally, conjunct by conjunct, each of the alternatives will have dynamic access to the pairs that come before it. That is, at a given input context, each branch of the nondeterministic output of the first conjunct will map some variable to a book that John read. These potential outputs 
will feed into the second conjunct independently, where they will again be supplemented nondeterministically with a variable mapped to a book that Mary read. Then once more for Fred. Every conjunct reads in a partially constructed map, augments that map with a new point, and then passes it on to the next conjunct.

This incrementality also unlocks the door to quantifier-internal anaphora (Brasoveanu 2011). Just as in ordinary sequences of sentences, indefinites in previous conjuncts can antecede anaphors in later conjuncts. In particular, the book that John is (nondeterministically) paired with can influence the possible book-witnesses for Mary and Fred. In this manner, a sentence like (7), repeated in (43a), is reduced to the sequence of updates in (43b). As a result, internal readings of comparative adjectives are reduced to special cases of external readings. They are explained by whatever anaphoric mechanisms generally allow them to target comparates in the broader context of evaluation. It's just that here, since the universal evaluates the conjuncts in series rather than in parallel, the context accumulates information throughout the evaluation. A sketch of the resulting composite update is given in (43c). The contribution of different is to prevent each successive update from assigning a variable to an object already assigned to a previous variable (an object in the image 'Im' of the previous assignment function).

(43) a. Every student read a different book.

[repeated from (7)]

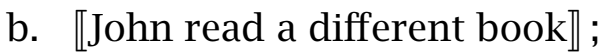

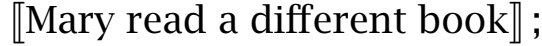

$\llbracket$ Fred read a different book》

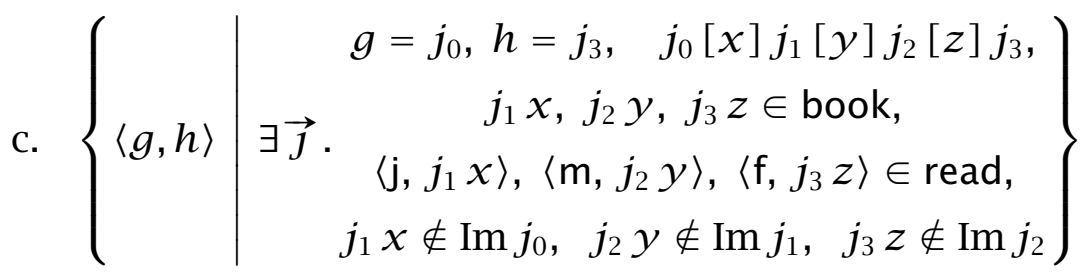

Incrementality additionally anticipates the possibility of order-sensitive quantification. Unlike classical conjunction, dynamic conjunction is not symmetric. $[A ; B]$ does not necessarily denote the same composite update as $[B ; A]$, since one of the conjuncts may be sensitive to properties of the context that the other alters. This means that the order in which the elements from the restrictor are evaluated with respect to the property defined by 
Incremental quantification

the nuclear scope can in principle make a difference to the overall truth conditions or felicity of the global update.

For sentences like (43a), this isn't the case because different is symmetric. If there is any injective, readership-preserving assignment of books to students, then the sequence of nondeterministic updates indicated in (43b) will find it among the many branching alternatives. But for sentences like (44a), the order of evaluation is crucial to the truth conditions, and this is where the incremental update strategy really shines. Under the assumption that naturally- or contextually-ordered restrictors are evaluated in their natural or contextual order, the analysis of (44a) proceeds in exactly the same fashion as the analysis of (43a) and (42a). The years divide the sentence into individual propositions, and the interest is compounded annually. That is, every year updates the input context with a book that Mary wrote in that year, and so long as at least one branch of the nondeterministic update results in an authorship-preserving function that increases monotonically in interestingness, the global update will succeed.

(44) a. Every year Mary wrote a more interesting book.

b. «In 2011 Mary wrote a more interesting book》;

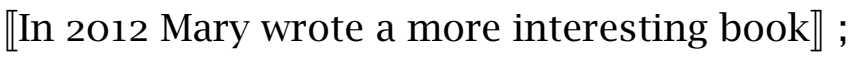

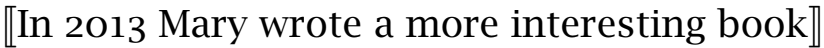

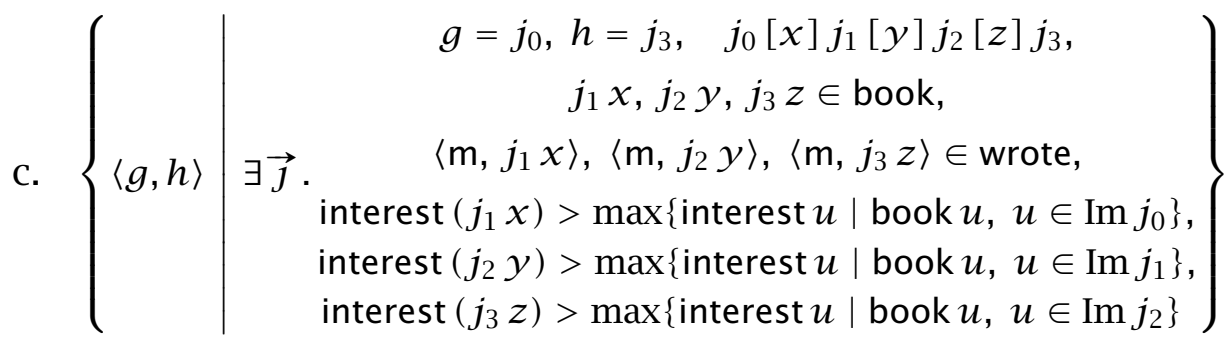

The data in Section 2.1 indicate that overt distributive universal quantifiers, and they alone, license internal readings of comparative adjectives, whether symmetric like different or asymmetric like more interesting. The only difference between the two is that the order of evaluation matters for sentences containing the latter, in the sense that different evaluation orders can result in different truth conditions. For that reason, such sentences are only felicitous in contexts in which the intended order of evaluation is apparent or given by world knowledge.

The incremental semantics sketched above offers a general, uniform explanation for the shared distribution of symmetric and asymmetric internal 
readings. And in the next section I'll show that it also explains the surprising wide-scope effects on display in Schlenker sentences, as well as the felicity of pair-list answers to universal questions. Then in Section 6, I'll review alternative analyses of the various phenomena, and show that in comparison, the current approach is both more general and more empirically adequate.

\section{Universal quantification as iterated dynamic conjunction}

As discussed in Section 3.1, pair-lists arise any time nondeterministic semantic objects are "conjoined" pointwise, whether this conjunction amounts to relation composition, pointwise fusion, or pointwise intersection. And since this is how conjunction is modeled in a variety of dynamic and alternativeoriented frameworks, there are in principle many options for formalizing the analysis.

However, Brasoveanu (2011) argues on the basis of typological evidence that internal readings of comparative adjectives are essentially tied to their general anaphoric potential. For this reason, it will be useful to work in an explicitly dynamic theory, since there are no provisions in current versions of Alternative or Inquisitive Semantics for sub-clausal and cross-sentential anaphora.

Even so, there are a variety of dynamic systems on the market. For current purposes, Charlow's (2014) recent grammar offers two advantages: (i) input and output contexts are modeled by simple lists of discourse referents, rather than total assignment functions (see also Vermeulen 1993, Dekker 1994, Eijck 2001, de Groote 2006, among others); and (ii) the semantics keeps track of both truth-conditional content and context-change potential, instead of attempting to recover the former from the latter. The first feature protects against destructive updates, and sets the incremental accumulation of pairs in dramatic relief. The second plays nice with a variety of scopeshifting mechanisms, something that is not often of interest in dynamic treatments, but will prove essential when we get to Schlenker sentences in Section 5.3. (Additional motivations for maintaining anaphoric potential alongside classical content are discussed extensively in Charlow 2014; cf. also Zeevat 1989 and Vermeulen 1995.) Of course, the reader is encouraged to recast the analysis in whatever dynamic terms he or she prefers. 
Incremental quantification

\subsection{A basic dynamic fragment}

In many dynamic setups, sentences denote relations over contexts. A sentence $\phi$ is considered true with respect to a model and an input context $i$ if it leads to at least one successful update; that is, if the relation $\llbracket \phi \rrbracket$ restricted to $i$ in its first coordinate is not empty (i.e., $\exists j .\langle i, j\rangle \in \llbracket \phi \rrbracket$ ).

In Charlow 2014, the basic propositional type contains a little more structure. ${ }^{8}$ Instead of mapping input contexts directly to sets of output contexts, sentences map input contexts to a set of output contexts paired with truth values (or, intensionally, propositions). Letting $\sigma$ designate the type of discourse contexts, this gives $\sigma \rightarrow\{t \times \sigma\}$ as the basic propositional type. The sentence John left, for instance, will denote the tagged update $\lambda s .\{\langle l e f t \mathrm{j}, s \cdot j\rangle\}$, which maps every input stack $s$ to a set containing a single pair. The pair represents a copy of the input stack extended with $j$, and either truth or falsity, depending on whether John left or not.

A nondeterministic update will behave as it always does in dynamic fragments, by sending input contexts to a potential multiplicity of output contexts. But here, each output context will in addition be paired with a truth value. So $A$ man left will denote the update $\lambda s$. \{ lleft $x, s \cdot x\rangle \mid \operatorname{man} x$, which maps an input stack $s$ to a set containing as many pairs as there are men, each of which maintains a copy of the input stack extended with a particular man $x$, and a truth value, determined by the truth or falsity of that man's having left.

More generally, every lexical item will map its input context to a set that pairs semantic values with output contexts. For example, the type of proper names will be $\sigma \rightarrow\{e \times \sigma\}$, the type of intransitive verbs will be $\sigma \rightarrow\{(e \rightarrow t) \times \sigma\}$, the type of intersective adjectives $\sigma \rightarrow\{((e \rightarrow t) \rightarrow e \rightarrow t) \times \sigma\}$, and so on. To give an impression of what we are heading toward, (45) provides a few example phrases, along with the types and denotations that a parser might assign them. In the coming sections, we will see how such denotations are assembled compositionally from the lexical entries and combinators afforded by the grammar.

8 Charlow's fragment is actually pervasively continuized, in the spirit of Barker 2002. This gives him an analytic handle on scope islands and paves the way for a unified treatment of the exceptional scope and binding behavior of indefinites and disjunctions. See Bumford 2013 for a continuized version of this analysis and a reference implementation in Haskell. 
Dylan Bumford

(45) \begin{tabular}{lll} 
Phrase & Type & Denotation \\
\cline { 2 - 3 } John & $\sigma \rightarrow\{e \times \sigma\}$ & $\lambda s \cdot\{\langle\mathrm{j}, s \cdot \mathrm{j}\rangle\}$ \\
a book & $\sigma \rightarrow\{e \times \sigma\}$ & $\lambda s \cdot\{\langle x, s \cdot x\rangle \mid$ book $x\}$ \\
read & $\sigma \rightarrow\{(e \rightarrow e \rightarrow t) \times \sigma\}$ & $\lambda s \cdot\{\langle\operatorname{read}, s\rangle\}$ \\
read a book & $\sigma \rightarrow\{(e \rightarrow t) \times \sigma\}$ & $\lambda s \cdot\{\langle\operatorname{read} x, s \cdot x\rangle \mid$ book $x\}$ \\
John read a book & $\sigma \rightarrow\{t \times \sigma\}$ & $\lambda s \cdot\{\langle\operatorname{read} x \mathrm{j}, s \cdot \mathrm{j} \cdot x\rangle \mid \operatorname{book} x\}$
\end{tabular}

Two things are worth noting about this sort of grammar. First, the dynamic machinations are maintained in lock-step with the basic truth-conditional content. Second, the grammar is pervasively dynamic, in the sense that every word gets a look at the state of the discourse at the moment it enters the semantic composition, and every word has an opportunity to manipulate that context before it is passed on. Given this, we can meaningfully speak of the dynamic component of the grammar, as something that wraps snugly and uniformly around the underlying model-theoretic interface.

The price to pay for this uniformity is a slightly more complicated notion of semantic composition. Since every word now denotes a dynamic update, we need a way of sequencing arguments into their functions, and we need the sequencing operation to apply pointwise in the case of nondeterministic updates. Here's the idea, assuming the constituent on the left is the underlying argument to the constituent on the right: (i) run the left constituent at the input stack $s$; (ii) stash each resulting semantic value $x$ and output stack $s^{\prime}$; (iii) one at a time, take the $\left\langle x, s^{\prime}\right\rangle$ pairs and pass them into the right constituent; (iv) collect the results. The definitions in (46) spell this process out formally.

\begin{tabular}{lll} 
(46) Combinator & Type & Denotation \\
\hline$\eta$ & $\alpha \rightarrow \mathbb{D}_{\alpha}$ & $\eta x=\lambda s \cdot\{\langle x, s\rangle\}$ \\
$\star$ & $\mathbb{D}_{\alpha} \rightarrow\left(\alpha \rightarrow \mathbb{D}_{\beta}\right) \rightarrow \mathbb{D}_{\beta}$ & $m \star k=\lambda s . \bigcup\left\{k x s^{\prime} \mid\left\langle x, s^{\prime}\right\rangle \in m s\right\}$ \\
$\backslash$ & $\mathbb{D}_{\alpha} \rightarrow \mathbb{D}_{\alpha \rightarrow \beta} \rightarrow \mathbb{D}_{\beta}$ & $L \backslash R=L \star(\lambda x . R \star(\lambda f . \eta(f x)))$ \\
$/$ & $\mathbb{D}_{\alpha \rightarrow \beta} \rightarrow \mathbb{D}_{\alpha} \rightarrow \mathbb{D}_{\beta}$ & $L / R=L \star(\lambda f . R \star(\lambda x . \eta(f x)))$
\end{tabular}

$\mathbb{D}_{\alpha}$ abbreviates $\sigma \rightarrow\{\alpha \times \sigma\}$. The $\eta$ and $\star$ operators break the combinatorial process into manageable conceptual bites. ${ }^{9}$ The former embeds traditional semantic values in an effectless dynamic data structure - that is, a function

9 They also define what's sometimes called a parser monad in the functional programming literature (e.g., Wadler 1994), or the State.Set monad in Charlow 2014. See Shan 2001 and Charlow 2014, part 1 for discussion of monads in natural language semantics. 
Incremental quantification

that (i) returns the traditional semantic value, and (ii) passes its input context straight through as output context.

The latter is more interesting. One way to conceptualize $m \star k$ is as a dynamically-aware method of function application. Instead of passing an argument directly into a function, the $\star$ first evaluates the argument $m$ at an input context, and then passes the underlying values into the function along with whatever modifications to the context they generate. The left and right slashes are dynamic analogs of the traditional categorial slashes. They additionally guarantee that discourse referents always flow from left to right, regardless of whether the argument precedes or follows the function.

This suffices to derive basic sentences of the Mary kissed John variety, as well as their nondeterministic variants (47). The latter passes each boy into the compositional context in a separate computational thread. So locally, within each value-stack pair, a boy might as well be 'John'. As in Alternative Semantics, the indeterminacy about just which boy actually plays the role of Mary's kissee is in some sense external to the core compositional process. This makes it possible to interpret the indefinite DP in situ.

(47) a. Mary kissed John.

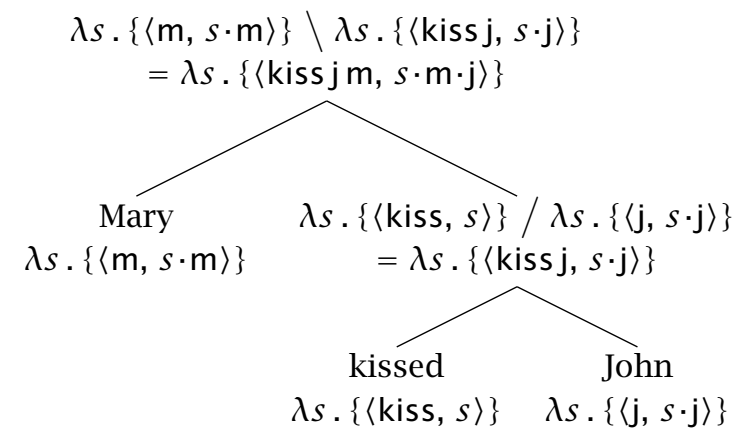

b. Mary kissed a boy.

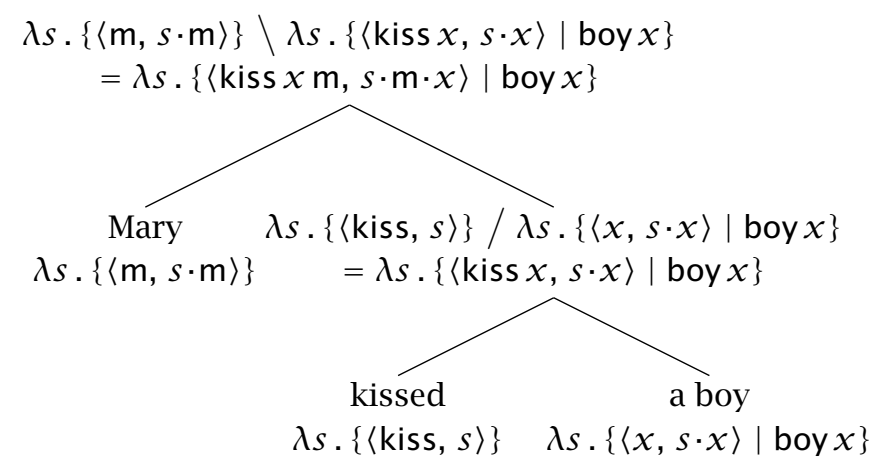


The semantics also already has a handle on dynamic conjunction and pronominal reference. Dynamic conjunction is in fact just conjunction "lifted" into the dynamic grammar, in the same way that $\llbracket \mathrm{read} \rrbracket=\eta \mathrm{read}=\lambda s$. $\{\langle\mathrm{read}, s\rangle\}$ is just a dynamicization of the $e \rightarrow e \rightarrow t$ relation read. That is, the grammar already feeds discourse information from left to right through every meaningful component of the sentence, so all that conjunction per se has to worry about is conjoining the values inside of its arguments. The binding takes care of itself.

Pronouns are equally simple. A pronoun is like any other deterministic noun in that it reads in an input context and returns an individual and an output context. What makes a pronoun a pronoun is that the individual it returns depends on which individuals are lying around in the input context. For simplicity, I'll assume that pronouns are indexed to particular stack coordinates, so that $h e_{1}$, for instance, picks out the most recent individual on its input stack. The denotations of and and he are laid out in (48), and applied to an example in (49).

(48) Item Type Denotation

$\begin{array}{lll}\text { and } & \mathbb{D}_{t \rightarrow t \rightarrow t} & \lambda s \cdot\{\langle\lambda p q \cdot q \wedge p, s\rangle\} \\ \text { he } & \mathbb{D}_{e} & \lambda s .\left\{\left\langle s_{n}, s\right\rangle\right\}\end{array}$

(49) Mary kissed John and he sneezed.

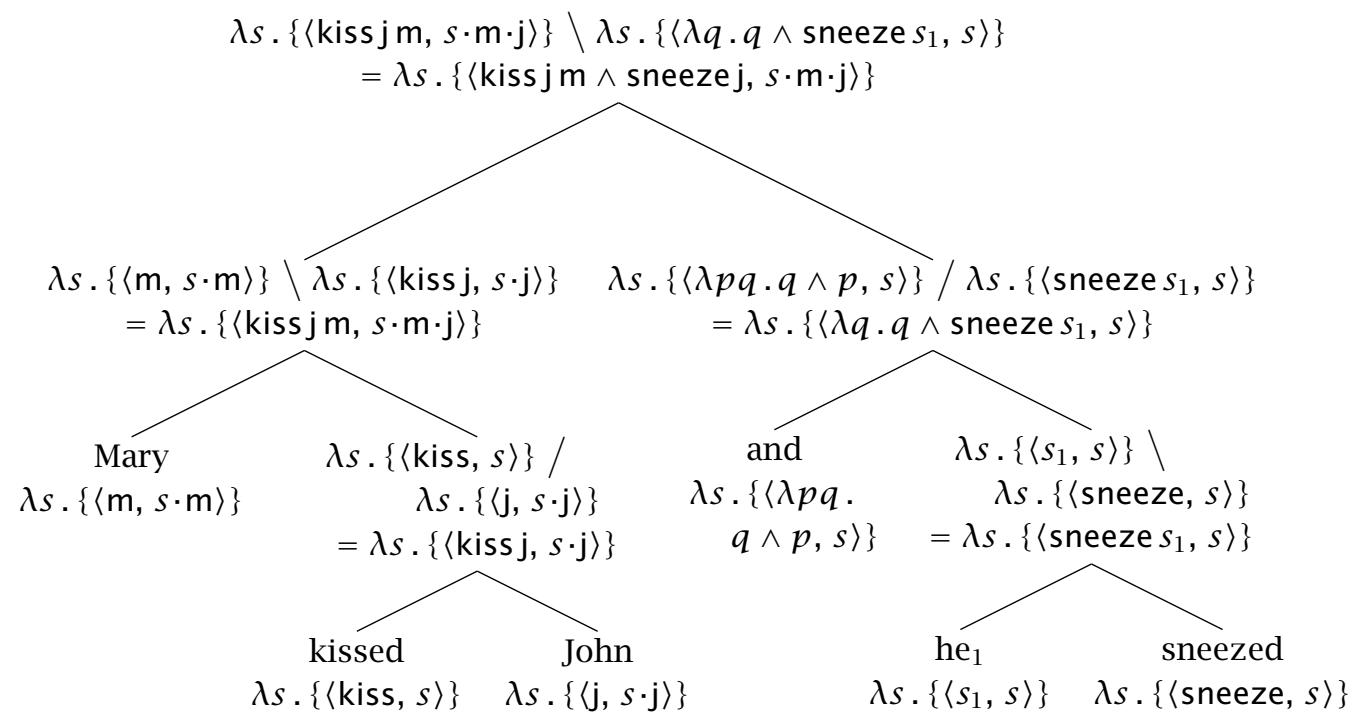


Incremental quantification

But the fragment so far is not prepared to handle genuine quantifiers of the sort that appear in the data of Section 2, much less inverse scope. For this, we need a notion of scope-taking.

\subsection{Scope shift and quantification}

\subsubsection{Quantifier raising}

In order for quantificational DPs to scope over their semantic contexts, they must at some point take an $e \rightarrow \mathbb{D}_{t}$ argument parameter representing the nuclear scope property that they ascribe to the individuals they quantify over. This creates a familiar type-clash that the slashes in (46) cannot resolve.

The standard solution to this problem is to appeal to a distinct interpretive mechanism for quantificational phrases, which are displaced from their surface syntactic positions. I will assume such a mechanism is operative here as well, though other means of scope-shift are possible. Displaced quantifier phrases will be interpreted according to the traditional QR rule in (5Oa), where $g$ is an assignment function keeping track of which argument slots belong to which quantifiers. Like everything else, traces are interpreted dynamically, as functions from input contexts to individuals and output contexts. Wherever possible, I will omit the assignment function from the $\llbracket \cdot \rrbracket$ notation.

(50) a. $\llbracket\left[\mathrm{QP}_{n} \beta\right] \rrbracket^{g}=\llbracket \mathrm{QP} \rrbracket^{g}\left(\lambda x \cdot \llbracket \beta \rrbracket^{g[n \mapsto x]}\right)$

b. $\llbracket t_{n} \rrbracket^{g}=\lambda s .\{\langle g n, s\rangle\}$

The entries in (52) give a few examples of the sorts of denotations we might assign to quantificational determiners. They rely on two further bits of notation, defined in (51), that will be helpful when talking about restrictors and nuclear scopes. First, the corners ${ }^{r} \cdot{ }^{\urcorner}$convert dynamic properties (type $\mathbb{D}_{e \rightarrow t}$ ) to sets of dynamic individuals (type $\left\{\mathbb{D}_{e}\right\}$ ), in the same way that characteristic sets convert vanilla properties to sets of vanilla individuals. For instance, 'boy' picks out the set $\{\lambda s .\{\langle x, s\rangle\} \mid$ boy $x\}$, which simply wraps each boy up in an input-output structure. ${ }^{10}$

10 The procedure for converting dynamic properties to sets of dynamic individuals is complicated by the possibility of properties with their own anaphoric restrictors (e.g., boy that she likes), as well as properties with their own dynamic side effects (e.g., farmer who owns a donkey). In order to handle such cases smoothly, the operation should actually behave as in (i), which evaluates the restrictor $P$ at the same input as the determiner it restricts, 
Second, the $\operatorname{TRUE}_{s}$ operator reifies the usual dynamic notion of truth: TRUE $_{s}$ holds of a dynamic proposition $\alpha$ (type $\mathbb{D}_{t}$ ) just in case updating $\alpha$ with the input context $s$ yields at least one truthful output value. For instance, the proposition $\lambda s .\{\langle\mathrm{F}, s\rangle,\langle\mathrm{T}, s\rangle\}$ is TRUE at any input $s$; the proposition $\lambda s .\{\langle\mathrm{F}, s\rangle,\langle\mathrm{F}, s\rangle\}$ is not TRUE at any input $s$; and $\lambda s .\left\{\left\langle\right.\right.$ left $\left.\left.s_{1}, s\right\rangle\right\}$ is TRUE at any input $s$ whose last (most recent) coordinate left.

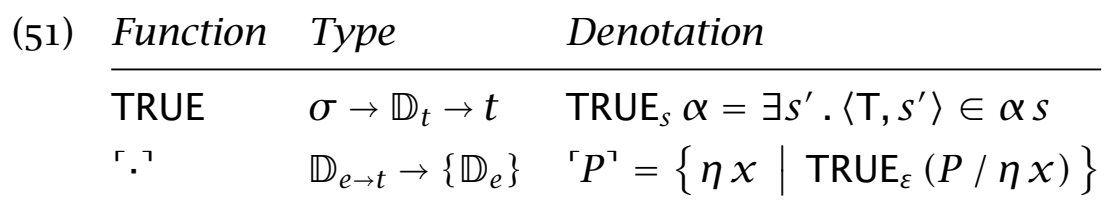

With these pieces in place, we may suppose that at least one, once supplied with a restrictor $P$ and nuclear scope $k$, denotes the deterministic, externally static update that is TRUE if at least one (dynamic) individual in $\left.{ }^{r} P\right\urcorner$ leads to a successful update when passed through $k$. Similarly, no $P$ denotes the deterministic, static update that is TRUE whenever none of the individuals in $\left.{ }^{\ulcorner} P\right\urcorner$ can be successfully sequenced into $k$.

In contrast, the non-quantificational determiners the, $a$, and two do not by their nature take scope. Instead, they convert their restrictors into dynamic individuals, and pass those individuals along into the compositional stream. The definite article returns the state-sensitive mereological sum of those entities that satisfy the requirements of its restrictor. The singular indefinite article spins each of those entities into a distinct compositional thread. As seen in (47), these threads will run in parallel for the future of the computation. Thus the "individual" that it returns is not only stateful (and thus potentially dynamic), but also nondeterministic. The cardinal determiner two is also nondeterministic, but where $a$ runs a parallel computation for every individual in $P$, two runs parallel computations for every pair of individuals in $P$.

and bottles each to-be-quantified-over individual up with any potential discourse referents it introduces.

(i) ${ }^{\mathrm{r}} P^{\urcorner s}=\left\{\lambda s^{\prime \prime} .\left\{\left\langle x, s^{\prime \prime}+\left(s^{\prime}-s\right)\right\rangle \mid\left\langle\mathrm{T}, s^{\prime}\right\rangle \in(P / \eta x) s\right\} \mid \operatorname{TRUE}_{s}(P / \eta x)\right\}$ 
Incremental quantification

\begin{tabular}{|c|c|c|}
\hline Item & Tуре & Denotation \\
\hline at least one & $\mathbb{D}_{e \rightarrow t} \rightarrow\left(e \rightarrow \mathbb{D}_{t}\right) \rightarrow \mathbb{D}_{t}$ & $\lambda P k s . \eta\left(\exists m:{ }^{r} P^{\urcorner} \cdot \operatorname{TRUE}_{s}(m \star k)\right) s$ \\
\hline no & $\mathbb{D}_{e \rightarrow t} \rightarrow\left(e \rightarrow \mathbb{D}_{t}\right) \rightarrow \mathbb{D}_{t}$ & $\lambda P k s . \eta\left(\neg \exists m:{ }^{\ulcorner} P^{\urcorner} \cdot \operatorname{TRUE}_{s}(m \star k)\right) s$ \\
\hline (all) the & $\mathbb{D}_{e \rightarrow t} \rightarrow \mathbb{D}_{e}$ & $\begin{array}{l}\lambda P s . \boxplus^{\top} P^{\urcorner} s, \\
\quad \text { where } m \boxplus m^{\prime} \equiv m \backslash\left(\eta \oplus / m^{\prime}\right)\end{array}$ \\
\hline some/a & $\mathbb{D}_{e \rightarrow t} \rightarrow \mathbb{D}_{e}$ & $\lambda P s . \bigcup\left\{m s \mid m \in{ }^{r} P^{\urcorner}\right\}$ \\
\hline two & $\mathbb{D}_{e \rightarrow t} \rightarrow \mathbb{D}_{e}$ & $\lambda P s . \bigcup\left\{\left(m \boxplus m^{\prime}\right) s \mid m, m^{\prime} \in{ }^{\ulcorner} P^{\urcorner}\right\}$ \\
\hline
\end{tabular}

The tree in (53) provides an example derivation with both quantification and scope-taking.

(53) Mary kissed at least one boy.

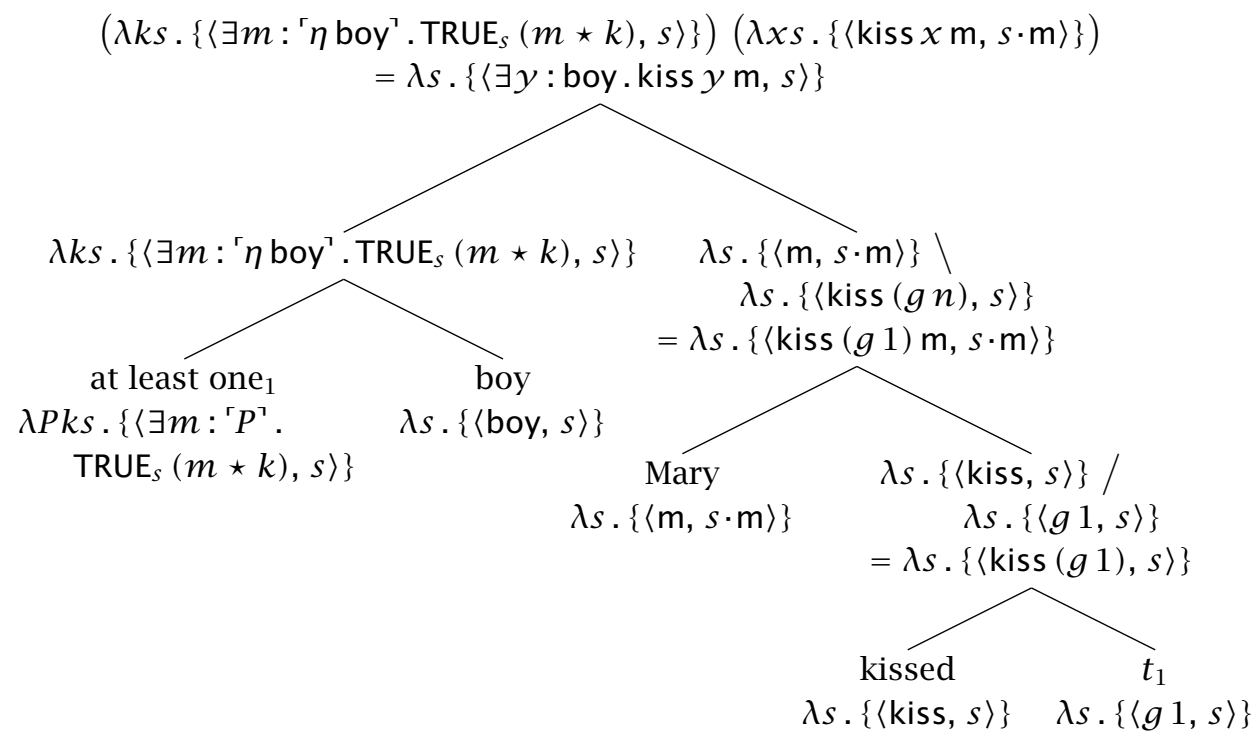

\subsubsection{Binding and type shifting}

The fragment still needs two basic functions in order to account for the data in Section 2. First is a mechanism for "lifting" dynamic individuals into dynamic generalized quantifiers. The strategy will be the same here as it is in familiar Montagovian frameworks (e.g., Partee 1986): $\uparrow$ converts an individual into a quantifier by abstracting over its possible continuations. The only 
Dylan Bumford

difference is that in the dynamic setting, "argumental" semantic objects need to be sequenced into their continuations. ${ }^{11}$

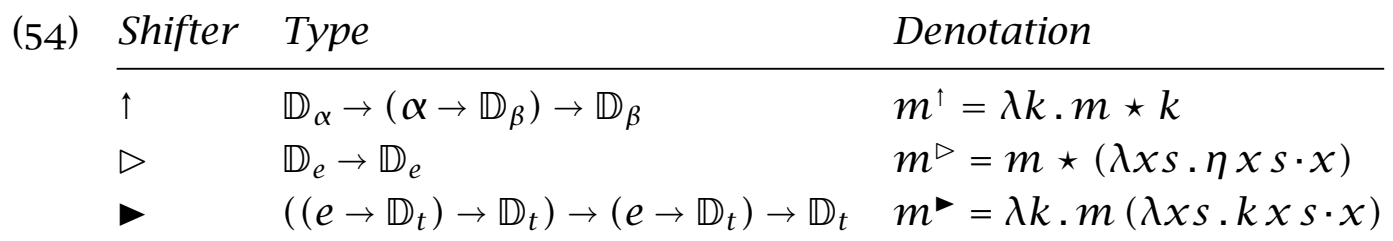

Second, we need a way to actually update contexts! Charlow (2014) relegates the introduction of discourse referents to the pair of combinators $\square$ and $\triangleright$. The former, $\triangleright$, converts a stateful (but side-effect-free) individual into a full-throated dynamic entity that leaves its mark on the context. It works just as well with singleton individuals like 'John' (【John $\left.\rrbracket^{\triangleright}=\lambda s .\{\langle\mathrm{j}, s \cdot \mathrm{j}\rangle\}\right)$ as it does with nondeterministic individuals like $a$ boy $\left(\llbracket\right.$ a boy $\rrbracket^{\triangleright}=\lambda s .\{\langle x, s \cdot x\rangle \mid$ boy $x$ \}). The latter, lifts this side-effecting action into the GQ type. The only difference between a generalized quantifier $m$ and its context-updating analog $m \bullet$ is that $m \bullet$ takes care to evaluate its nuclear scope $k$ in a context supplemented with $x$, the individual it is currently quantifying over. ${ }^{12}$

The benefit of this is that it allows us to dissociate the essential quantificational semantics of determiners from the potential dynamic effects they may have on contexts. In this framework, only the former is part of the lexical meaning of a quantifier; the latter is regulated by the plumbing of the grammar. So rather than speaking of particular determiners as if they were inherently (temporarily) context-updating, we may speak of particular occurrences of determiners as context-updating or not, as the occasion demands. Importantly, this does not affect their capacity to quantify properties over individuals, only their ability to bind pronouns in their arguments. In Section 6, I will suggest an opportunity to exploit this modularization of semantic force and dynamic potential.

11 Note that just as Montague's lifting operation is $\eta$-equivalent to (reverse) function application, $\uparrow$ is $\eta$-equivalent to the sequencing applicator $\star$, which is the dynamic analog of function application in this monadic grammar. This is not an accident, and the interested reader is referred to Charlow 2014 for further discussion of the relationship between scope-taking and program combination.

12 As one might hope, there is a nice relationship between $\triangleright$, $\triangleright$, and $\uparrow$, namely $\left(m^{\triangleright}\right)^{\dagger}=\left(m^{\dagger}\right)$. That is, dynamically charging $m$ and then lifting the result is equivalent to lifting $m$ and then charging the result. In fact, we technically only need $\triangleright$, as $m^{\triangleright}=\left(m^{\dagger}\right) \downarrow \eta$. 
Incremental quantification

\subsubsection{Universal quantification}

The true quantifiers defined in (52) have in common that the elements of their restrictors are evaluated against their nuclear scopes independently. For example, to calculate whether or not the sentence No boy left is true, the semantics first isolates each of the boys, and then for each one checks to see whether or not he left. If any of those tests deliver a successful update, the entire sentence fails; otherwise it succeeds.

This isolating behavior is precisely what prevents internal readings of comparative adjectives. The sentence No boy read a different book asks of each boy whether or not he read a different book. The adjective can't possibly compare the books read by different boys because the boys are evaluated in parallel. Even as flexible as it is, different can't index books in alternative quantum states of the update. Each thread has only its own history for comparison.

But because distributive universals simply generalize dynamic conjunction, the sentence Every boy read a different book will evaluate the boys in series. As the conjuncts accumulate, one boy's books determine another boy's "different books", ad iteratum. This impression is cashed out technically in (55). The grand semicolon folds Boolean conjunction over the alternatives derived by passing the elements of the restrictor into the nuclear scope $k$. (I will return to the covert distributivity operator $\Delta$ shortly.)

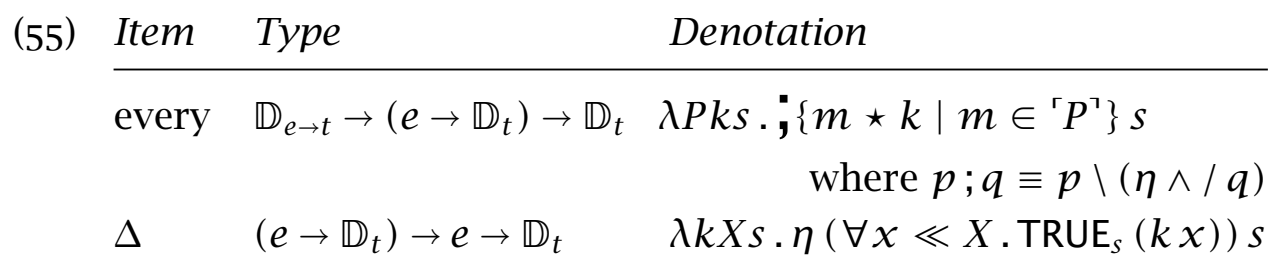

When the scope of every contains an indefinite or disjunction, the conjuncts formed by feeding in the elements of the restrictor will all be nondeterministic. This is the situation sketched in (42). At a given input, the composite update denoted by the sentence Every student read a book will generate a great variety of outputs. Each potential output will correspond to a pairing of students with books; its resulting semantic value will be $T$ if that particular pairing is a subset of the read relation; and the entire sentence will be TRUE just in case one of the possible pairings results in a semantic value of $\mathrm{T}$. 
(56) Every student read a book.

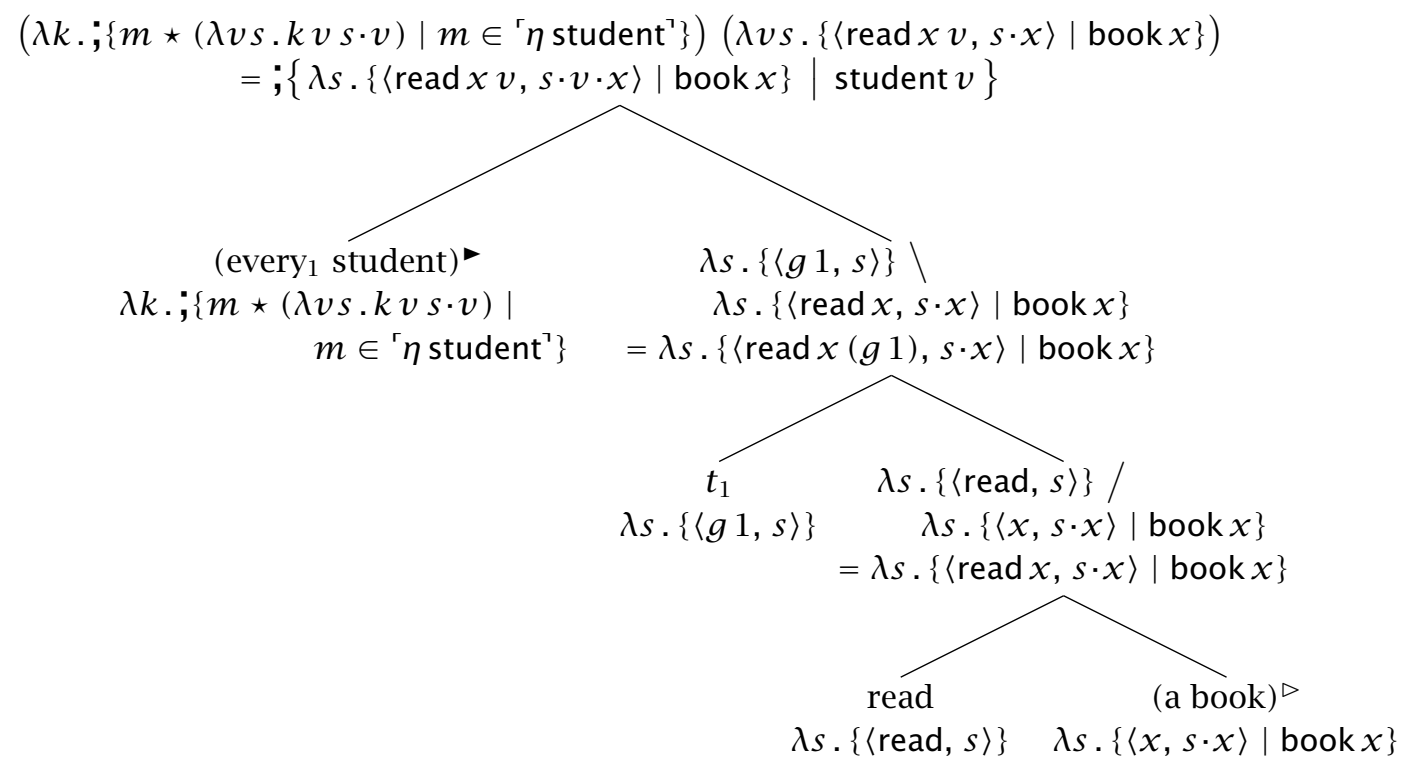

As promised, the final expression at the top of the tree, repeated in (57a), is equivalent to the iterated conjunction in (57b), assuming John, Mary, and Fred are the students. The iterated conjunction, in turn, reduces to the update in (57c). Every potential output adds to its input a list of individuals alternating between students and books, and returns truth as its semantic value if and only if all the students read their corresponding books.

(57) a. $;\{\lambda s .\{\langle\operatorname{read} x v, s \cdot v \cdot x\rangle \mid \operatorname{book} x\} \mid$ student $v\}$

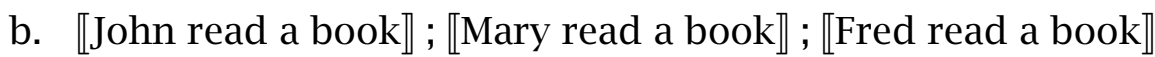

c. $\lambda s .\{\langle\operatorname{read} x \mathrm{j} \wedge \operatorname{read} y \mathrm{~m} \wedge \operatorname{read} z \mathrm{f}, s \cdot \mathrm{j} \cdot x \cdot \mathrm{m} \cdot y \cdot \mathrm{f} \cdot z\rangle \mid x, y, z \in \operatorname{book}\}$

For completeness, I have provided a covert distributivity operator $\Delta$ designed to make sense of sentences like (58), when understood to convey that 6 invitations in total were extended. There is considerable controversy regarding the availability and distribution of such readings, as discussed by Dotlačil (2010, Chapter 2), who also provides experimental evidence for their marginal status. But for the sake of discussion, I assume, following Link (1987), Roberts (1987), Schwarzschild (1996), Winter (2001), and many others, that distributive readings, when available, are due to a covert adverbial operator that universally quantifies a predicate over the atoms of some plurality. 
Incremental quantification

(58) Three boys invited two girls.

[Gil 1982, (7a)]

In the case of (58), $\Delta$ converts the verb phrase invited two girls from a property of singular individuals - satisfied by those who invited two girls - into a property of sum individuals - satisfied by those whose atomic members invited two girls apiece. But there is an important difference between $\Delta$ and every: only the latter is incremental. The covert operator, on the other hand, is much closer to the standard sort of entry assigned to distributive determiners in dynamic frameworks. Like the earlier quantifiers at least one and no, but unlike the lexical universal every, the covert operator $\Delta$ evaluates the individuals it distributes over independently. As before, this will spread the components of the update too thin for internal adjectives to compare them.

Also like those earlier quantifiers, but unlike every, the covert distributor genuinely quantifies over the possibilities opened up in its nuclear scope. For each atomic member $x$ of its restrictor $X, \Delta$ checks whether or not $x$ leads to a successful update when passed through the property denoted by its scope $k$; that is, it checks that at least one of the updates in $k x$ is successful. As discussed in Section 3.1, dynamic conjunction does not standardly force this sort of Boolean inspection. For instance, the DPL conjunction defined in (37) does not test its assignments to see if they both yield truth; it composes the updates point by point, implicitly concatenating the requirements imposed by the left conjunct with those imposed by the right. In this fragment, $\Delta$ generalizes the former quantificational behavior; every generalizes the latter, nondeterministic behavior. The distinction will play a role in the account of arbitrary functional readings in Section 5.3.

Readers familiar with dynamic approaches will also likely notice that there is another respect in which every differs from $\Delta$, at least one, no, and other genuine quantifiers. As defined in (55), every is not only internally dynamic, but also externally dynamic, in the sense that discourse referents introduced in its scope remain available for anaphors that are not.

On the one hand, this predicts that singular individuals that play a role inside the universal computation should be able to bind pronouns outside that computation. That is, we should be able to follow (56) up with a sentence like He liked it to mean that one of the boys liked one of the books (depending on how the pronouns are resolved). Of course this is not at all possible in discourse, which suggests that individual referents should not survive the scope of the universal. 
On the other hand, if anything at all in natural language is externally dynamic, it is sentential conjunction. After all, the sensibility of $A$ man walked in; he whistled is the raison d'être of dynamic semantics. Yet, He liked it is no better a continuation to the sequence of conjuncts in (57b) than it is to (56). The problem, I contend, is not that every is hardwired to cover its own dynamic tracks, but rather that singular pronouns are pragmatically unresolvable when used after a string of equally-good referential targets. ${ }^{13}$

In any case, it would not change the analysis presented here if every were brought in line with the other externally static quantificational determiners. For example, the hypothetical determiner $\llbracket$ every $^{\prime} \rrbracket=\lambda P k s .\left\{\langle p, s\rangle \mid\left\langle p, s^{\prime}\right\rangle \in\right.$ $\llbracket$ every $\mathbb{P} k s\}$ behaves exactly like every except that at the end of the computation, it replaces the output stack of every alternative with the input stack, effectively wiping out referents local to the universal (see Brasoveanu 2008, p.151 for a backtracking protocol along these lines). Crucially, this sort of stack erasure would not interfere with the internal dynamics of iterative quantification. Everything inside of the universal's "loop" would still have access to the referents accumulated up to that point in the evaluation. Nor would it alter the resulting pointwise alternative structure at the heart of pair-list questions and Schlenker truth conditions, as we'll see in the next section.

But first, before we leave this discussion of the difference between every and $\Delta$, let me point out that there is no theoretical reason that the covert distributivity operator should be quantificational, rather than compositional. It could just as easily have been defined as in (59) below, playing an adverbial counterpart to the iterating semantics of the lexical universal. The reason for defining it as I have in (55) is empirical. According to the generalizations of the literature summarized in Section 2.4, $\Delta$ does not support any of

13 That said, one of the reviewers observes a contrast between (ia) and (ib) below, which suggests that even when a pronoun could in principle target a particular antecedent from the restrictor of a universal (by dint of its $\phi$-features), it refuses to do so. In my view, and I believe the reviewer's, this is a nice illustration of the fact that what counts as a "good" referential target is complicated by factors beyond what is technically available in the semantic context.

(i) a. \#Each member of No Doubt had a solo career, but only she was successful.

b. Tony had a solo career; Gwen had a solo career; and Tom had a solo career. But only she was successful. 
Incremental quantification

the pair-list phenomena under discussion, despite its distributive universal quantificational force.

$$
\llbracket \Delta^{\prime} \rrbracket=\lambda k X s . ;\{\eta x \star k \mid x \ll X\} s
$$

However, recall from footnote 4 the recent hypothesis explored in Dotlačil 2010 and Brasoveanu \& Dotlačil 2012. They argue that the difficulty of internal readings in the scope of plurals is merely a reflection of these determiners' general aversion to covert distributivity operators in the first place. If this is right, then we are free to opt for (59) and eschew the stipulative distinction between covert and overt distributors. Then, as with the internal adjectives, we would derive the absence of (or perhaps dispreference for) pair-list readings in questions like (21) and conditionals like (33) as a result of the plural DPs' general hostility toward $\Delta^{\prime}$.

\section{Applications to pair-list phenomena}

\subsection{Internal adjectives}

Since the grammar largely takes care of the dynamic legwork required for anaphora, it is easy to give meanings for comparative adjectives. The meaning of different book, for example, according to the definitions in (6o), is a dynamic property true of any book not already on the input stack. Similarly, bigger book picks out the set of books that are bigger than any other books mentioned so far.

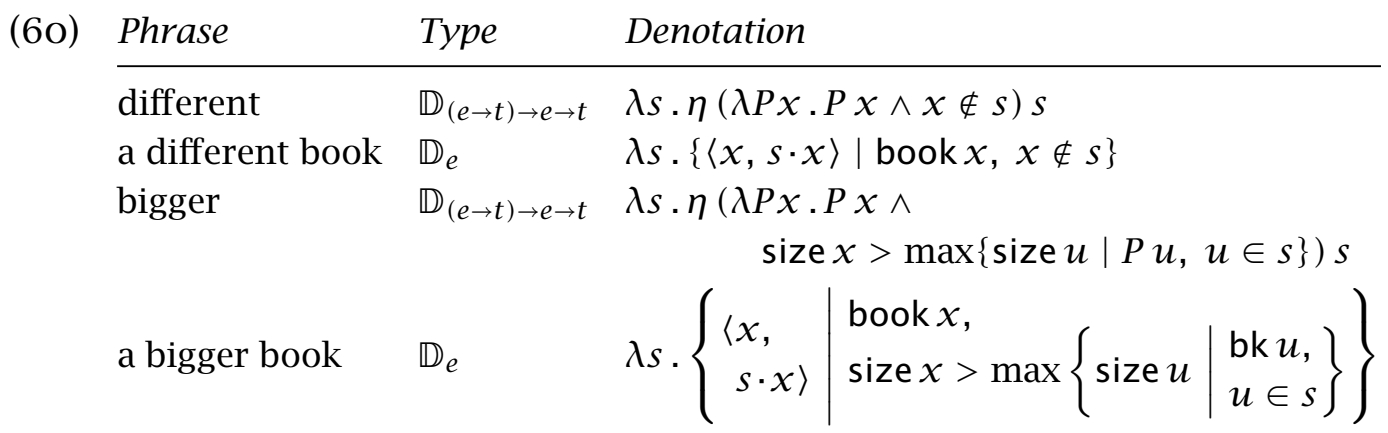

With these definitions in hand, the derivation of (61) is minimally different from that of (56). The only thing it adds to (56) is a constraint on the possible witnesses for each boy's book: because of the word different, the indefinite is additionally restricted to just those objects that have not previously appeared in the discourse. 
(61) Every student read a different book

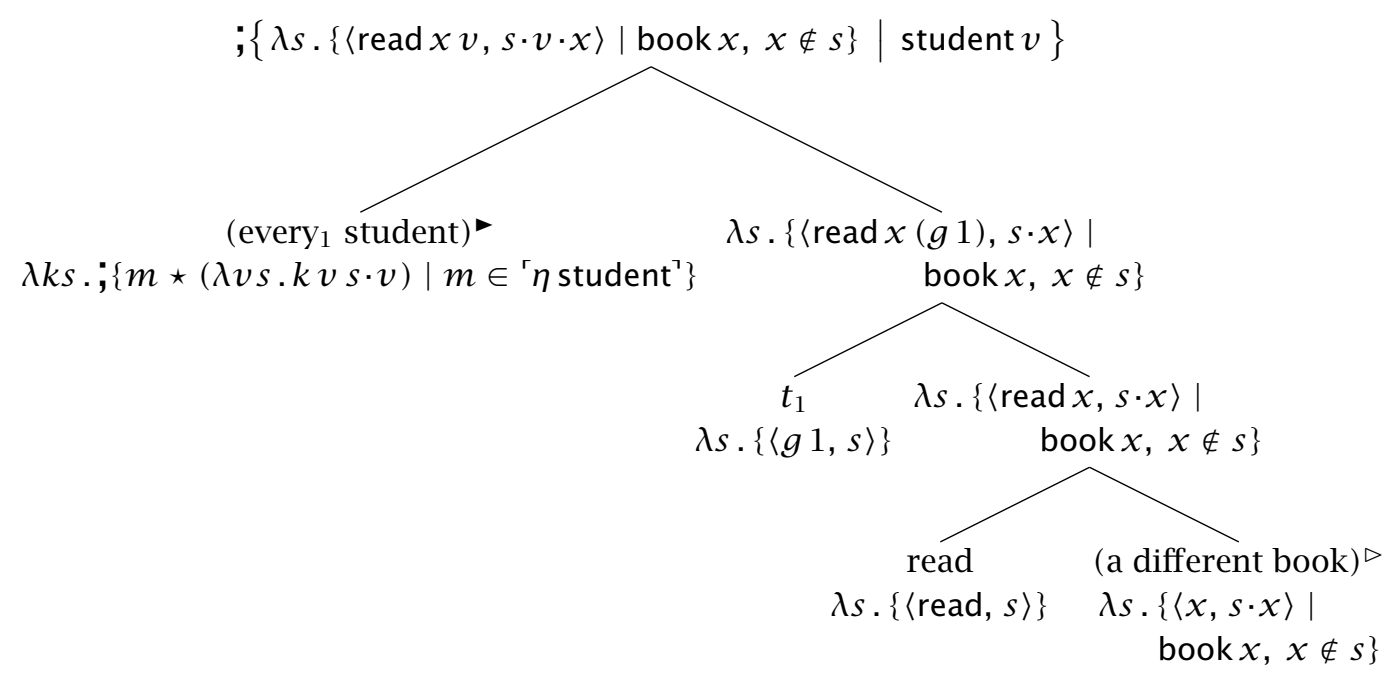

As before, the final expression at the root of (61) is equivalent to the iterated conjunction in (62b), which in turn reduces to (62c). Because the update denoted by (62) is incremental, the $x \notin s$ condition becomes more difficult to satisfy as the computation progresses. In each nondeterministic thread, John's book limits Mary's options, and then Mary's book further limits the options for Fred.
a. $;\{\lambda s .\{\langle\operatorname{read} x v, s \cdot v \cdot x\rangle \mid \operatorname{book} x, x \notin s\} \mid$ student $v\}$

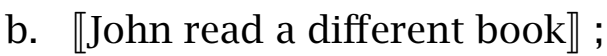
$\llbracket$ Mary read a different book》;
$\llbracket$ Fred read a different book》

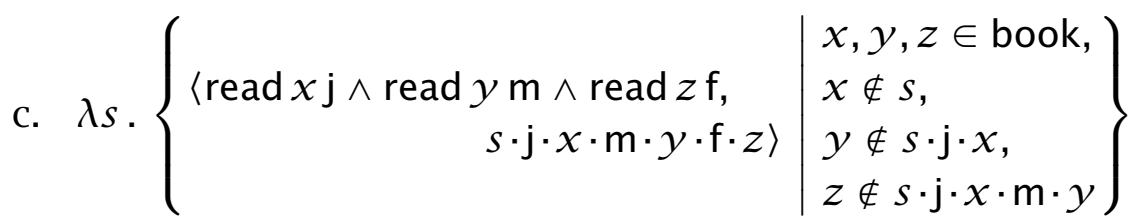

A point of concern: the denotations in (6o) are far too blunt to capture the range of anaphors that comparative adjectives can target. Many external uses of different, like the one in (63a), clearly target specific stack indices, in the same way that anaphoric pronouns do, rather than the entire discourse history. Similarly, internal uses of different typically ignore items on the stack prior to the first pass through the universal loop that they sit in. For instance, the cooperative learning groups of (63b) should presumably be free 
Incremental quantification

to read Frog and Toad Are Friends even though it was mentioned earlier in the discourse. Even more thornily, the boys under investigation in (63c) very likely named each other as perpetrators, a scenario the denotation in (6o) could not countenance, given that the early namers could not serve as later namees.

(63) a. ${ }^{\gamma}$ We recommend that you read the best-selling The Complete Idiot's Guide to Getting Published. We're not saying this because two of us wrote it, either. In fact, we don't care if you read a different book (yeah, right!).

b. ${ }^{\gamma}$ To introduce students to Chapter Titles and Summaries, you might choose to read aloud "A Lost Button" from Frog and Toad Are Friends. [...] Another alternative is to give each cooperative learning group a different book that has chapter titles.

c. ${ }^{\gamma}$ The fact that none of these stories were consistent and that each boy named a different boy as the primary perpetrator apparently did not trouble the police or the prosecution.

These examples, and many others like them, demand a more flexible, underspecified lexical entry for different. I will not attempt to provide anything like a comprehensive account of how different hones in on its comparates in context, but I will point out some formal resources for dealing with this flexibility. First, recall that whether or not a particular DP contributes a referent to the stack depends on whether it was type-shifted (with $\triangleright$ or $\triangleright$ ) to do so. Thus, DPs that nobody ever refers to again, like the book of (63b)'s first sentence, need never be added to the stack in the first place, in which case they will not interfere with subsequent stack queries.

Second, we should almost certainly allow for particular instances of different to target particular (not necessarily contiguous) subregions of the input stack. The different of (63a), for example, is better represented by a denotation along the lines of $\lambda s .\left\{\left\langle\lambda P x . P x \wedge x \notin\left[s_{i}\right], s\right\rangle\right\}$, where $i$ is the stack index of the idiot's guide.

Finally, though the use of plain stacks as a model of linguistic state keeps the basic formal system lightweight and manageable, it makes for an extremely impoverished representation of semantic context. Partly for this reason, many dynamic systems run on top of much more complex data structures. Nouwen (2003, 2007), for example, building on work by van den Berg (1996b), deploys a set of stacks together with a labeling function for highlight- 
ing specific indices associated with grammatical positions in the sentence. So the state represented by (64) might be one in which the first column contains a set of students and the second a set of books that those students read (stackwise). Additionally, the labels $x_{s}$ and $x_{o}$ indicate that the columns they adorn were generated by DPs in subject and object positions, respectively (see Nouwen 2007 for details). Using something as simple as this labeling function, we could orient the different in (63c) toward the accumulating list of named boys (who will all be object-labeled), simultaneously ignoring the boys doing the naming (who will be subject-labeled). We could accomplish the same thing without a labeling function by restricting the purview of different to every other boy on the stack, thereby implicitly targeting just the referents added by object-position DPs.

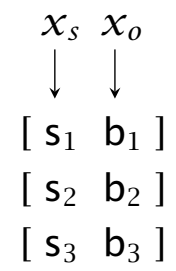

Unfortunately, I have nothing to say about how to identify on a particular occasion of use the relevant subspace of semantic context that a speaker intends to target when using the word different. Admittedly, allowing for the lexical semantics of comparative adjectives to ignore arbitrary elements of the stack will lead to formally possible resolution strategies that no listener would ever consider, but this problem is not unique to anaphoric adjectives. In natural discourse, the potential referents of pronouns already are highly constrained by all sorts of pragmatic factors that semantic systems do not capture. Likewise, I assume that in trying to make sense of a particular instance of different in context, listeners will choose a subset of the context according to pragmatic heuristics that are well beyond the scope of this paper.

That said, because any syntactic instance of an adjective will in the end receive exactly one semantic resolution (just as any particular instance of a singular pronoun will receive exactly one semantic index), the grammar will at least ensure that all the students of (61) are held to the same standard. If John is evaluated with respect to whether or not he read a book different from the book at index 4, then so is Mary and so is Fred. This would generate an external reading of (61). Alternatively, if John is evaluated with respect to whether or not he read a book different from anything mentioned so far, 
Incremental quantification

then so is Mary and so is Fred. This would generate an internal reading of (61), as in (62). But there will never be a reading that requires some students to read a book different from Frog and Toad Are Friends and others to read a book different from The Complete Idiot's Guide to Getting Published, nor a reading that evaluates some students on external grounds (did they read a book different from Frog and Toad Are Friends?) and others on internal grounds (did they read a book different from each other? did they read a book different what the first guy read? etc.). This much, at least, is semantics.

\subsection{Pair-list questions}

The denotation of a question is often identified with the set of propositions that resolve it (Hamblin 1958, Karttunen 1977). For example, according to Hamblin $(1958,1973)$, the question in (65) should denote the set of alternatives in (65a), one alternative for each of its possible answers - that is, for each possible boy that Mary might have kissed.

(65) Which boy did Mary kiss?
a. $\{$ kiss $x \mathrm{~m} \mid$ boy $x\}$
b. $\lambda s .\{\langle$ iss $x \mathrm{~m}, s \cdot x \cdot \mathrm{m}\rangle \mid$ boy $x\}$

[cf. (47)]

This denotation bears a close resemblance to the nondeterministic update shown in (65b), which represents the meaning of the corresponding indefinite declarative Mary kissed a boy. Most recent incarnations of the Hamblin tradition embrace this correspondence. Many, in fact, explicitly unify the formal representations of interrogatives, indefinite declaratives, and disjunctions (e.g., Kratzer \& Shimoyama 2002, Alonso-Ovalle 2006, Mascarenhas 2009, Groenendijk \& Roelofsen 2009, Ciardelli, Groenendijk \& Roelofsen 2013). The principal insight from these research programs is that even though wh-words generally signal a different kind of pragmatic move than indefinite determiners or disjunction particles do, the two kinds of expressions ultimately make the same contribution to the compositional semantics: they both introduce alternatives.

The current fragment differs from standard Alternative and Inquisitive Semantic approaches in that it is dynamic, but the underlying alternative bone structure is exactly the same; whatever role inquisitiveness plays in Inquisitive Semantics, nondeterminism plays here. (See Charlow 2014, Section 5.5 for further discussion of the connection between dynamic semantics 
and alternative-oriented semantics.) Working backward then, the right way to represent constituent questions like (65) in this dynamic system is to recycle the denotations of the corresponding indefinite declaratives. In the simplest case, we can even identify the lexical semantics of which with that of $a$. As a result, setting syntactic issues aside, it is immediately clear that $\llbracket(65) \rrbracket=(65 \mathrm{~b})$.

More to the point, taking the Inquisitive leap and setting $\llbracket$ which $\rrbracket=\llbracket a \rrbracket$ predicts the acceptability of pair-list answers to universal questions without any ado. Again, pretending that English is a wh-in-situ language, the question in (66) composes exactly as in (56) and yields the same denotation, repeated below.

(66) Which book did every student read?

(67) a. $;\{\lambda s .\{\langle\operatorname{read} x v, s \cdot v \cdot x\rangle \mid \operatorname{book} x\} \mid$ student $v\}$

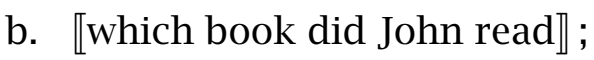

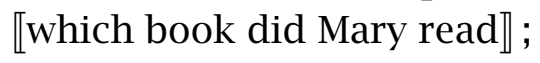

«which book did Fred read》

c. $\lambda s .\{\langle\operatorname{read} x \mathrm{j} \wedge \operatorname{read} y \mathrm{~m} \wedge \operatorname{read} z \mathrm{f}, s \cdot \mathrm{j} \cdot x \cdot \mathrm{m} \cdot y \cdot \mathrm{f} \cdot z\rangle \mid x, y, z \in$ book $\}$

As before, the wide-scoping universal sequences the alternatives in its scope. Here, those alternatives are the questions depicted in (67b). The final denotation is the (state-sensitive) set of propositions each of which declares for some triplet of books $x, y$, and $z$, that John read $x$, Mary read $y$, and Fred read $z$. Construed inquisitively, this is the question whose answers all provide for each student a book that that student read.

This analysis of pair-list answers, which reduces a universal question to the sequence of its component sub-questions, converges with that of Krifka (2001), who approaches the issue from the perspective of composite speech acts more generally. He observes that all speech acts, including questions, can be performed in sequence, and in that sense may be "conjoined". So according to Krifka, for any two speech acts $\mathcal{A}$ and $\mathcal{B}$ of the same type, we should understand the conjunction $\mathcal{A} \& \mathcal{B}$ as denoting the composite act comprised of a performance of $\mathcal{A}$ followed by a performance of $\mathcal{B}$. For instance, we understand the consecutive acts in (68) as asking composite questions, imposing composite commitments, expressing composite opinions, etc. Of course in every case, how we technically model a "composite act" will depend on the nature of the formal object that we take to represent the individual 
Incremental quantification

sub-acts. But at least in the case of questions and assertions - modeled here as potentially nondeterministic updates - it is entirely natural to implement speech-act composition as relation composition (i.e., ';'). ${ }^{14}$

(68) a. Which dish did Al make? And which dish did Bill make?

b. Eat the chicken soup! And drink the hot tea!

c. How beautiful this is! And how peaceful! [Krifka 2001, (43)]

With this in mind, Krifka conjectures that when a distributive universal quantifies into a speech act, it does so by forging a sort of performance pipeline composed of the acts in its scope (69). Executing that composite act amounts to executing, in sequence, all of the individual acts in the chain. Clearly the denotation for every provided in (55) is an instantiation of this general schema ( $a$ here is Krifka's type for speech acts).

\begin{tabular}{lll} 
Item & Type & Denotation \\
\hline every & $(e \rightarrow t) \rightarrow(e \rightarrow a) \rightarrow a$ & $\lambda P Q x . \&\{Q x \mid P x\}$
\end{tabular} [Krifka 2001, (82)]

But what of the other quantificational operators? Recall from Section 2.2 that only overt universal distributors successfully quantify into questions. In particular, according to Krifka (1992), Srivastav (1992), and Gawron \& Kehler (2003), singular questions with definite plurals and DP conjunctions do not accept pair-list answers. For instance, Krifka judges that (7ob) is not a felicitous answer to (70).

(70) Which movie did the boys rent last night?

a. (The boys rented) $Z$.

b. \#Al rented $A$, Bill rented $B$, and Carl rented $C$. [Krifka 1992, (8b)]

Given the common assumption that pair-list answers are what happens when a question is interpreted in the scope of something distributive (see, e.g., Groenendijk \& Stokhof 1984, Chapter 3), one might have expected that (7ob) would be an appropriate response to the $p l>\Delta>$ wh reading of (70a), sketched in (71a). ${ }^{15}$ However, as constructed in (55), $\Delta$ is inherently quan-

14 Krifka actually gestures toward a quasi-game-theoretic analysis in terms of conversational moves and responses that is intended to generalize across all types of speech acts.

15 Technically, $\Delta$ 's scope here is parasitic, in the sense of Barker 2007: its first argument is the continuation of the DP that it distributes over. This is avoidable, but not without the sort of scopal acrobatics better managed by continuized grammars (cf. Charlow 2014, Section 4.5). 
tificational; it evaluates the atoms in its restrictor with respect to whether or not they pass truthfully through its nuclear scope. If that nuclear scope is a function from individuals to questions, then this testing operation will presumably be undefined, since questions are just not the sorts of things that can be true or false.

This is admittedly a little tricky, since on the one hand I'd like to assimilate the representations of indefinite declaratives and interrogatives (following Kratzer \& Shimoyama 2002, Groenendijk \& Roelofsen 2009, etc.), but on the other hand, I'd like to reserve the right to define a truth predicate that only applies to declarative updates. Adjudicating between this rock and this hard place is beyond the scope of this paper, ${ }^{16}$ but note that even if we allow TRUE to apply to the relevant updates inside of $\Delta$, the denotation assigned to (71a) will end up equivalent to (71b). This function is just the deterministic update that tests a context to make sure that every boy rented at least one movie. Or in Inquisitive terms, this is the non-inquisitive proposition whose only possibility is the set of worlds in which every boy rented at least one movie. It is certainly not a pair-list question, and arguably not a question at all.

(71) a. $\llbracket\left[\left(\text { the }_{1} \text { boys }\right)^{\dagger}\left[\Delta\left[\right.\right.\right.$ which movie did $t_{1}$ rent $\left.\left.]\right]\right] \rrbracket$ $=\left(\lambda k \cdot \boxplus^{\ulcorner} \eta\right.$ boys $\left.^{\urcorner} \star k\right)\left(\Delta\left(\lambda v \cdot \llbracket\right.\right.$ which movie did $t_{1}$ rent $\left.\left.\rrbracket^{g[1 \mapsto v]}\right)\right)$ $=\left(\lambda k \cdot \boxplus^{\ulcorner} \eta\right.$ boys $\left.^{\urcorner} \star k\right)$ $\left(\lambda X s . \eta\left(\forall x \ll X . \mathrm{TRUE}_{s} \llbracket\right.\right.$ which movie did $t_{1}$ rent $\left.\left.\rrbracket^{g[1-x]}\right) s\right)$

b. $\llbracket\left[\left(\text { the }_{1} \text { boys }\right)^{\dagger}\left[\Delta\left[t_{1}\right.\right.\right.$ rented a movie $\left.\left.]\right]\right] \rrbracket$ $=\left(\lambda k . \boxplus\left\ulcorner\eta\right.\right.$ boys $\left.{ }^{\urcorner} \star k\right)$

$\left(\lambda X s . \eta\left(\forall x \ll X . \mathrm{TRUE}_{s} \llbracket t_{1}\right.\right.$ rented a movie $\left.\left.\rrbracket^{g[1-x]}\right) s\right)$

$=\eta(\forall x \ll \oplus$ boy. $\exists y:$ movie. rent $y x)$

$=\lambda s \cdot\{\langle\forall x \ll \oplus$ boy $. \exists y:$ movie. rent $y x, s\rangle\}$

The other quantificational determiners in (52) will have the same problem. Because they demand truth of their nuclear scopes, they will either fail to quantify into a question, or in so doing, destroy it. This, however, leaves open the possibility that other non-quantificational operators could outscope $w h$ words, including disjunctions, other indefinite DPs, and any lifted constants,

16 One possibility is to reify the type constructor $\mathbb{D}_{\alpha}$ that currently abbreviates $\sigma \rightarrow\{\alpha \times \sigma\}$, and to create an isomorphic but distinct constructor for questions $\mathbb{Q}_{\alpha}$. Then the types of the lexicon could be systematically relaxed to accept both $\mathbb{D}_{\alpha}$ and $\mathbb{Q}_{\alpha}$ arguments, except for TRUE, which would continue to accept only $e \rightarrow \mathbb{D}_{t}$ continuations. 
Incremental quantification

though the resulting so-called "choice readings" of questions are controversial (e.g., Belnap 1982, Groenendijk \& Stokhof 1984, Szabolcsi 1997, Krifka 2001).

\subsection{Schlenker readings}

Taking the derivation in (56) and (57) as a model, the sentence in (72a) will denote the dynamic proposition described by $(72 b)$. The interesting thing about (72b), over and above the fact that it was constructed incrementally, is that it is itself nondeterministic. At a given input $s$, there are as many alternative outputs as there are functions from students to areas.

(72) a. Each student improved in some area.

b. $\lambda s .\left\{\begin{array}{c|c}\langle\operatorname{imprv} x \mathrm{j} \wedge \operatorname{imprv} y \mathrm{~m} \wedge \operatorname{imprv} z \mathrm{f}, \\ s \cdot \mathrm{j} \cdot x \cdot \mathrm{m} \cdot y \cdot \mathrm{f} \cdot z\rangle & x, y, z \in \text { area }\end{array}\right\}$

The nondeterminism of (72) is important. Farkas (1981) argues that indefinite DPs, unlike genuinely quantificational DPs, are free to take scope over any subset of other logical elements in the sentences in which they are evaluated. Rooth \& Partee (1982) observe that disjunction and indefinites pattern alike with respect to this sort of exceptional scope-taking, in particular to the exclusion of conjunctions.

Charlow (2014) makes the case for tying the exceptional scope and binding properties of indefinites and disjunctions to their non-quantificational nature, in whatever form it takes. He argues that as far as scope-taking is concerned, there is no important difference between deterministic things like proper names - which everybody agrees are scopeless - and nondeterministic things like indefinites, except that the latter are potentially multiply realized. But each of those realizations is effectively quarantined in an independent branch of the composition, in which it looks to the grammar exactly like a constant. Really, the only things whose scope gets caught in the usual tensed-clause webs are items that not only generate alternatives, but also quantify over those alternatives.

Even in semantic frameworks without any notion of nondeterministic meanings, exceptional-scope interpretations of indefinites and disjunctions are sometimes chalked up to the same mechanisms (Rooth \& Partee 1982, Schlenker 2006, Slade 2011). If these approaches are correct in deriving the potential for exceptional scope from nondeterminism - or probably more accurately, from non-quantificationalism - then the meaning represented 
by (72b) should show the same scopal flexibility as garden variety names, disjunctions, and indefinites.

This is exactly what happens in Schlenker's example (31), repeated below in (73). The entire indeterminate distribution of areas to students takes scope over the conditional that contains it. Formally, this amounts to first lifting the antecedent clause, according to the polymorphic rule in (54), and then QRing the clause out into the matrix, as shown in (74).

(73) If each student makes progress in \{some, a (certain)\} area, nobody will flunk the exam. [repeated from (5)]

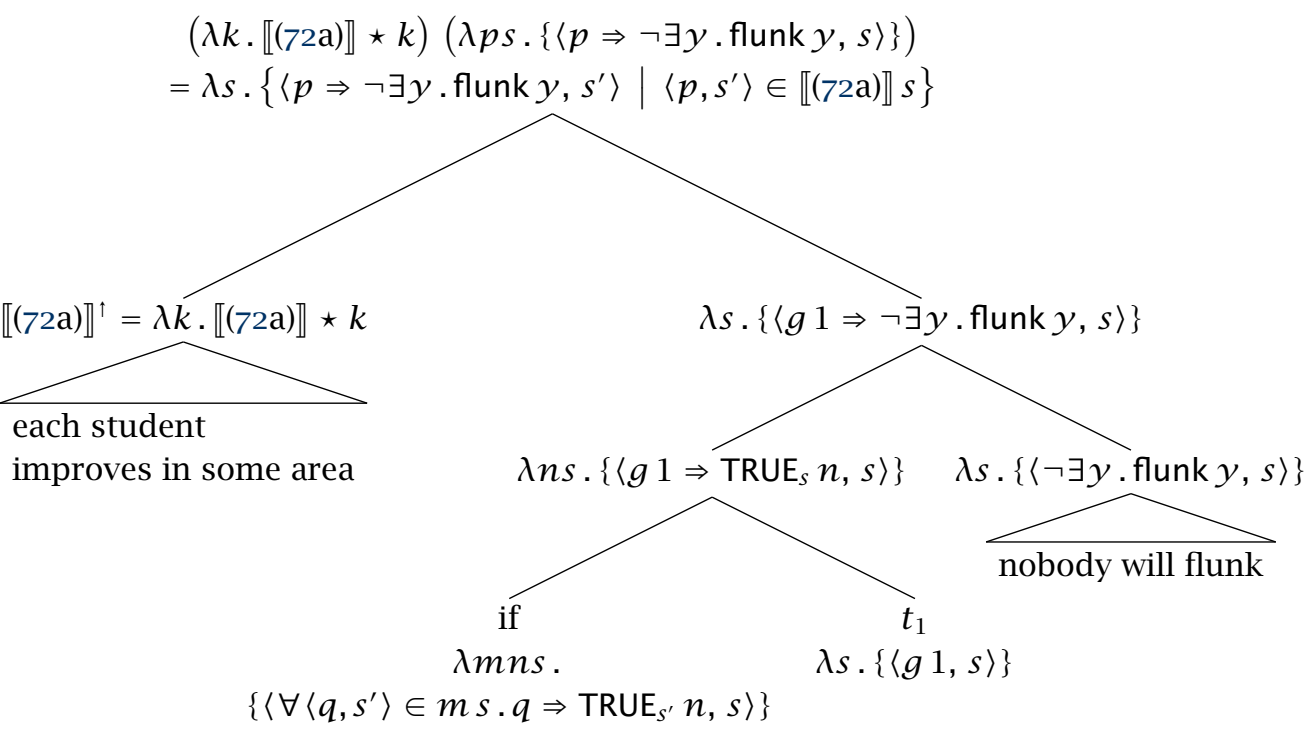

The root denotation is repeated in (75a). Setting aside the inputs and outputs, the interpretation of (73) delivers a set of conditionals, depicted in (75b), each of which declares that if John, Mary, and Fred all improve in particular areas, then nobody will flunk. The sentence is predicted to be true just in case for some trio of areas $x, y$, and $z$, John's improving in $x$, Mary's improving in $y$, and Fred's improving in $z$ are jointly sufficient to guarantee that nobody flunks. And this is exactly the sort of scenario Schlenker had in mind.

(75) a. $\lambda s .\left\{\left\langle p \Rightarrow \neg \exists y\right.\right.$. flunk $\left.\left.y, s^{\prime}\right\rangle \mid\left\langle p, s^{\prime}\right\rangle \in \llbracket(72 \mathrm{a}) \rrbracket s\right\}$

b. $\{\operatorname{imprv} x \mathrm{j} \wedge$ imprv $y \mathrm{~m} \wedge$ imprv $z \mathrm{f} \Rightarrow \neg \exists y$. flunk $y \mid x, y, z \in$ area $\}$ 
Incremental quantification

Unlike choice-functional accounts of functional readings, the semantics here makes no explicit appeal to function variables representing ways of pairing up students with subject matters. The "pair-list" truth conditions emerge here as a result of two processes. First, the iterated applications of dynamic conjunction over the set of propositions representing the students' potential improvements yield the alternatives in (72b). In and of itself, this is essentially a disjunctive-normal-form encoding of the atomic propositions implicit in (72a). But then these alternatives take scope over the conditional that embeds them, via the same independently motivated channels that generally support exceptional scope readings of disjunctions and indefinites (Charlow 2014, Part 2). Again, apart from dynamics, the resulting constraint on models is equivalent to the constraint imposed by existentially quantifying over Skolemized choice functions, as in (4), but here it arises just from the standard compositional interaction between quantification and scope-taking.

The empirical advantages of this approach are discussed in the next section. For now, notice that classically (non-iteratively) distributive quantifiers will not generate Schlenker readings. In particular, the covert distributor $\Delta$ defined in (55) will not support the super-conditional dependencies that every does, even when the antecedent clause takes widest scope. Compare (76a) and (76b).

(76) a. If every boy rents Psycho or Vertigo, then they'll all pass their exams.

b. If the boys rent Psycho or Vertigo, then they'll all pass their exams.

The former can describe a situation in which the boys' collective success depends on each of them renting the right movie (maybe the one his film professor thinks would be best for him, maybe the one he knows the most about, or maybe something much more random that the speaker is not in a position to articulate). But ( $76 \mathrm{~b})$ has no such reading. It might mean that one of those two movies is the key to success for everybody, if the disjunction takes scope over the conditional. Or if covert distributors are available in such an environment, it might mean that the boys will pass if they can all just manage to rent at least one of the two movies, whichever they feel like. This distributive reading of the antecedent corresponds to the parse in (77), interpreted in (77b).

(77) a. $\left[\left(\text { the }_{1} \text { boys }\right)^{\dagger}\left[\Delta\left[t_{1}\right.\right.\right.$ rent Psycho or Vertigo $\left.\left.]\right]\right]$

b. $\eta(\forall x \ll \oplus$ boy. $\exists y:\{$ psyc, vert $\}$. rent $y x)$ 


$$
=\lambda s .\{\langle\forall x \ll \oplus \text { boy. } \exists y:\{\text { psyc, vert }\} \text {. rent } y x, s\rangle\} \quad \text { [cf. (71b)] }
$$

But there is no wide-scoping pair-list reading analogous to (75). To see this, consider what happens when we drop (77a) in for the QRed antecedent of (74). The resulting denotation, shown in (78), is equivalent to the surfacescope if $>\Delta>$ or reading discussed above. This is because the update that (77a) denotes is deterministic. It consists of a single alternative, true if every boy rented at least one of the movies, false otherwise, and therefore behaves for all intents and purposes like a proper name of type $t$. And as is always the case with names and other constant values, QRing the entire sentence has no detectable effect on scope, because the lifted sentence just passes its denotation back into the remnant it has evacuated.

(78) $(\lambda k . \llbracket(77 \mathrm{a}) \rrbracket \star k)(\lambda p s .\{\langle p \Rightarrow \neg \exists y$. flunk $y, s\rangle\})$

$$
=\lambda s .\left\{\left\langle p \Rightarrow \neg \exists y \text {. flunk } y, s^{\prime}\right\rangle \mid\left\langle p, s^{\prime}\right\rangle \in \llbracket(77 \mathrm{a}) \rrbracket s\right\}
$$$$
=\lambda s .\{\langle(\forall x \ll \oplus \text { boy. } \exists y:\{\text { psyc, vert }\} \text {. rent } y x) \Rightarrow \neg \exists y \text {. flunk } y, s\rangle\}
$$

\section{Discussion and comparison to related work}

\subsection{Summary}

Before I attempt to place the analysis here in the context of other theories that cover some of the same empirical ground, let me briefly summarize. The proposal has been to construct the meaning of overt distributive universal quantifiers directly from the meaning of sentential conjunction. In a variety of popular semantic frameworks, the meanings of sentences are potentially indeterminate (i.e., inquisitive, alternative-denoting, multiply-verified, nondeterministic, etc.). As a result, sentential conjunction is usually defined in such a way that it conjoins the individual points (possibilities, alternatives, verifiers, etc.) within the sets denoted by its arguments. The resulting proposition is a more finely crosshatched picture of the world, one in which every cell witnesses the truth of the first conjunct in some particular way and witnesses the truth of the second conjunct in some particular way. The seeds of pair-lists are sewn right into these distributions over particulars.

If this is the right intuition, then pair-lists emerge completely organically from sentential conjunction in a number of semantic settings. For exactly the same reason, they also emerge from sentences with distributive universal 
Incremental quantification

quantifiers, provided that such quantifiers directly engage sentential conjunction. What's more, the emergent collection of finely-articulated alternatives is every bit as inquisitive or nondeterministic as an indefinite DP or disjunction. This predicts that universal sentences, when generating pair-lists, will exhibit the same exceptional scope and binding behavior as those more traditional sources of uncertainty.

To make these matters concrete, I presented a fragment in a recent dynamic framework that explicitly accounts for patterns of exceptional scopetaking. The distributive universal was defined so as to (i) construct a number of potential updates by mapping its nuclear scope over the individuals in its restrictor, and (ii) compose the updates in sequence. In addition to providing a particular implementation of the universal-as-generalized-conjunction idea, this incremental update procedure also predicts the possibility of quantifierinternal anaphora. This is because information accumulates over the course of the universal computation in such a way that discourse referents associated with elements of the universal's restrictor are available for anaphors associated with any elements evaluated subsequently, just as discourse referents generated in earlier conjuncts can bind pronouns in later conjuncts.

Finally, I argued that these predictions align with empirical generalizations already present in the linguistic literature, and explain patterns of readings that have proven a challenge for compositional semantics. Those generalizations, repeated from Section 2.4, are as follows:

i. An internal reading of a comparative adjective in a singular DP is only possible in the scope of a distributive universal quantifier (Beghelli 1997, Brasoveanu 2011).

ii. A pair-list answer to a singular wh-question is only felicitous when the $w h$-word is interpreted in the scope of a distributive universal quantifier (Szabolcsi 1997, Krifka 2001).

iii. An embedded clause containing a singular indefinite DP is only sensitive to an exceptionally-scoping pair-list association when the indefinite is interpreted in the scope of a distributive universal quantifier (Spector 2004, Schlenker 2006, Solomon 2011).

The availability of such readings in the presence of overt universal distributors follows immediately from the iterative semantics proposed here, together with current approaches to dynamic semantics and scope-taking, 
as demonstrated in Section 5. The unavailability of such readings in the presence of other quantifiers, including covert distributors - although see footnote 4 -follows from the standard, internally static, quantificational semantics they are typically assumed to have.

\subsection{Related work}

While I cannot do justice to the many nuanced theories of what pair-list/internal readings are or where they come from, I can make some general remarks about the types of alternative analyses that have been proposed. Recall that the question in (18), repeated in (79) has at least three superficially distinct types of answers, illustrated in (79a-c).

(79) Which woman does every man love?
a. Mary.
b. Alex loves Ann, Bill loves Bee, Carl loves Cat.
c. His mother.

[repeated from (18)]

There is a strong temptation to explain the pair-list answer in (79b) as a special case of the functional answer in (79). This sort of analysis is pursued in Engdahl 1986, Chierchia 1993, Dayal 1996, among others. Along similar lines, Schwarz 2001 argues that the exceptionally-scoping pair-list readings of conditionals like (80) arise from the same semantic processes that generate exceptionally-scoping functional readings of the Hintikkan variety (81). Chierchia (2001) and Schlenker $(1998,2006)$ also analyze examples like (80) in terms of existential quantification over functions, but where Schwarz takes the reading to exemplify an unusually arbitrary functional reading, Chierchia and Schlenker take it as evidence in favor of a more general treatment of indefinites as variables over (Skolemized) choice functions.

(80) If each student makes progress in \{some, a (certain)\} area, nobody will flunk the exam. [repeated from (31)]

(81) I know that every true Englishman adores a certain woman.

[repeated from (27)]

The difficulties for choice-functional treatments of indefinites are wellknown (see especially Geurts 2000, Schwarz 2001, Brasoveanu \& Farkas 
Incremental quantification

2011), so I won't rehearse them here. (See, e.g., Kratzer 1998, Winter 2001, Chierchia 2001, Schlenker 2006 for attempts to restrain and re-engineer choice functions to suit linguistic needs, and Brasoveanu \& Farkas 2011 and Charlow 2014 for arguments to abandon the effort and for alternative analyses of exceptional scope.)

But even when quantification over choice functions is restricted to the set of $e \rightarrow(e \rightarrow t) \rightarrow e$ mappings that are in some way linguistically or contextually natural/salient, the functional approach to pair-lists overgenerates "functional" readings in the scope of other quantifiers. Chierchia (1992) and many following him attribute the unavailability of pair-list answers to questions like (82) to the supposed unnaturalness of functions that map boys onto arbitrary dishes they didn't make. But such associations can become natural in context, which is presumably what accounts for the felicity of (83a). Yet, the question in (83b), which would be answered by exactly the same function that answers (83a), is a non sequitur.

(82) \#Which dish did no boy make?

[repeated from (19)]

(83) I know nobody turned in all of their assignments. So tell me, ...

a. which assignment did each student forget to turn in?

b. \#which assignment did no student turn in?

Similarly, as pointed out by Solomon (2011), the conditional in (84a) can be used to indicate that class-wide success is guaranteed by a particular distribution of improvements across students (Alex needs to improve in syntax, Bill in syntax, Carl in semantics, etc.). But the conditional in (84b) cannot be used to indicate that class-wide failure is guaranteed unless some student improves in the relevant area for him. It either means that either syntax or semantics is such that everyone will fail unless at least one student gets better at it, or it means that everyone will fail unless somebody gets better at something. This is surprising if the availability of arbitrary functional associations is determined by the naturalness of the association, since (84a) and $(84 \mathrm{~b})$ are justified by the same arbitrary pairing of students and subjects.

(84) a. If every student improves in syntax or semantics, no one will fail the exam.

$$
\begin{array}{r}
\exists f: \text { student } \rightarrow\{\text { syn, sem }\} .(\forall x: \text { student. improve }(f x) x) \Rightarrow \\
\neg \exists y \text {. flunk } y
\end{array}
$$


b. If no student improves in syntax or semantics, everyone will fail the exam.

$$
\begin{array}{r}
\# \exists f: \text { student } \rightarrow\{\text { syn, sem }\} .(\neg \exists x: \text { student . improve }(f x) x) \Rightarrow \\
\forall y \text {. flunk } y
\end{array}
$$

Groenendijk \& Stokhof (1989) offer an alternative explanation for the apparent restrictions on pair-list answers. For them, questions denote equivalence relations over worlds. A pair of worlds $\left\langle w, w^{\prime}\right\rangle$ will be in the denotation of a question $Q$ just in case $w$ and $w^{\prime}$ agree on the extension of the wh-DP's complement. The question Who left?, for example, will contain all $\left\langle w, w^{\prime}\right\rangle$ pairs such that left ${ }_{w}=$ left $_{w^{\prime}}$.

Wide-scoping quantifiers in questions contribute domain restrictions on relations, so that an interrogative like (79) denotes the partition $\lambda w w^{\prime}$. love $_{w}$ \man $=$ love $_{w^{\prime}} \backslash$ man (see Groenendijk \& Stokhof 1984, Chapter 6, Part 4 for details). This partition includes every pair of worlds that agree on the extension of the love relation restricted to men - that is, every pair of worlds that assign the same lovees to each man. The crucial requirement for deriving this denotation compositionally is that the quantificational DP every man has a unique witness set, namely the set of men.

Negative universal DPs like no man have no (nontrivial) witness sets, and cardinal DPs generally have more than one witness set, making them both unsuitable for wide-scope interpretations with respect to $w h$-abstracts. This is a good prediction, given the lack of pair-list readings for interrogatives like (82) and (85). However, definite plurals and conjunctions certainly do have unique witness sets, yet they too fail to support pair-list answers in singular wh-questions (see Section 2.2).

(85) a. \#Which man did more than two dogs bite?

b. ?Which man did two dogs bite? [Szabolcsi 1997, (29-30)]

Plurals and conjunctions are also a source of overgeneration for choicefunctional approaches to Schlenker sentences. Given that the antecedent of (86) can be interpreted distributively (in which case success is guaranteed as long as Alex and Bill each study at least one topic), it should be possible for the covert distributor to bind into a "natural function" (in which case there would have to be particular topics for each of Alex and Bill that guarantee success upon study). However, as reported in Spector 2004 and Solomon 2011 and discussed in Section 2.3, (86) has no such reading. 
Incremental quantification

(86) If Alex and Bill study some topic, the exam will be a success.

a. $\quad(\forall x \ll \mathrm{a} \oplus \mathrm{b} . \exists y$ : topic . study $y x) \Rightarrow$ success $\mathrm{e}$

b. $\# \exists f:\{\mathrm{a}, \mathrm{b}\} \rightarrow$ topic $(\forall x \ll \mathrm{a} \oplus \mathrm{b}$. study $(f x) x) \Rightarrow$ success e

[repeated from (33)]

Krifka (2001) provides yet another explanation for the emergence of pairlist answers to distributive universal questions. According to Krifka, the interrogative in (79) denotes the generalized conjunction of the questions formed by mapping the continuation of the universal DP over the universal's restrictor. As discussed in Section 5.2, the fragment I've developed in this paper can be seen as a compositional implementation of exactly this intuition. However, where Krifka's analysis is entirely silent on the connection between pair-list questions and pair-list readings of embedded clauses in declarative contexts, the present fragment demonstrates the manner in which both arise from iterated conjunction, together with a theory of exceptional scope.

Another prediction that Krifka mentions (pp. 13, 23), but does not take up, is that every should be underlyingly dynamic, given that speech act conjunction - which sits at the core of Krifka's universal - is not in general commutative. I have argued that this universal-internal dynamism is precisely what lays the groundwork for quantifier-internal readings of comparative adjectives, including asymmetric comparatives like taller. Because such adjectives are uniformly anaphoric (Brasoveanu 2011: p. 97), they are all capable of reaching back into previous conjuncts to find their comparates, as demonstrated in (87). Thus it comes as no surprise that such adjectives can also reach back into the history of a universal computation, since a universal computation, as I have defined it, is nothing more than a sequence of such conjunctions.

(87) Mary recited “The Raven”. Then Linus recited a different poem.

[Brasoveanu 2011, (1)]

The only other compositional attempt to derive internal readings of singular comparative DPs as a special case of their capacity for external readings comes from Brasoveanu (2011) (though see also Dotlačil 2010 and Bumford \& Barker 2013 for further developments and applications of his approach). Brasoveanu's analysis is based on two technical innovations. The first is a dynamic framework, Plural Compositional DRT (PCDRT), that builds on Muskens's (1996) CDRT, but replaces input and output assignment 
functions with input and output assignment matrices. The framework is developed extensively in Brasoveanu 2007, where it is used to model a variety of phenomena related to plural anaphora in subordination and donkey-type configurations.

Much like the stacks in (64), PCDRT is designed so that lexical relations will by default establish row-wise dependencies between discourse referents. The sentence in (88), for example, will relate an input matrix $I$ and an output matrix $J$ just in case $J$ is like $I$ except that its first column contains three boys and its second column three girls such that for each row $r, r_{1}$ invited $r_{2}$ (this is often called a cumulative reading). The update is schematically diagrammed below.

(88) Three $_{1}$ boys invited $\mathrm{two}_{2}$ girls.

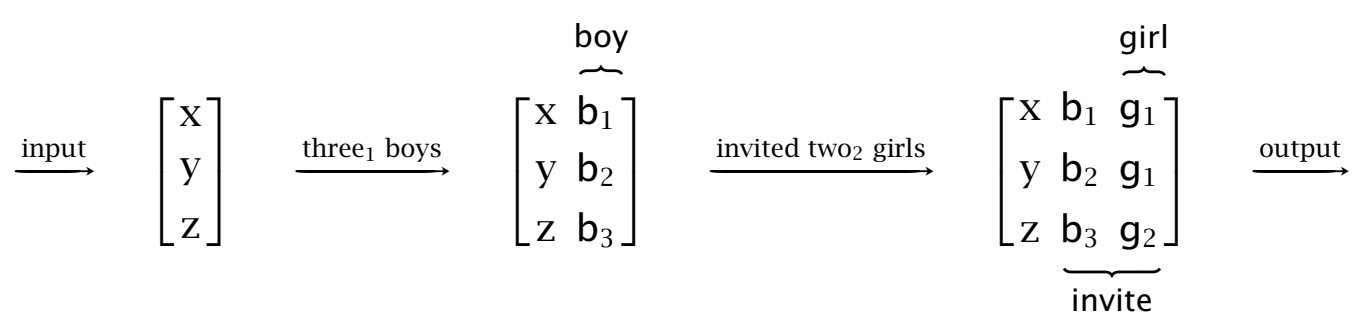

Two context matrices $G$ and $H$ are distributively related with respect to a property $P$ and an index $i$ iff for each referent $d$ in the $i$ th column of $G$, the two submatrices $G_{i=d}$ and $H_{i=d}$ are normally related with respect to $P d$ (for details and discussion of distributive operators along these lines, see e.g., van den Berg 1996a, Definition 5, Nouwen 2007, (32-37), Brasoveanu 2011, (245), Brasoveanu 2012, (173)). For example, the controversial surfacescope distributive reading of (88) might correspond to an update like the one sketched in (89). The subject DP requires that the first column of its output context contain in total a set of three distinct boys. Then the distributive operator $\delta_{1}$ requires that the second column of its output context contain two girls for each of those three boys in the first column, such that every boy invited the girl in his row.

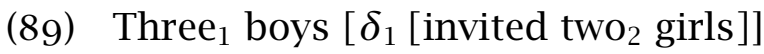


Incremental quantification

$$
\stackrel{\text { input }}{\longrightarrow}\left[\begin{array}{l}
x \\
y \\
z
\end{array}\right] \stackrel{\text { three }{ }_{1} \text { boys }}{\longrightarrow}\left[\begin{array}{ll}
x & b_{1} \\
y & b_{2} \\
z & b_{3}
\end{array}\right] \stackrel{\delta_{1} \text { [invited two }{ }_{2} \text { girls] }}{\longrightarrow}\left[\begin{array}{lll}
x & b_{1} & g_{1} \\
x & b_{1} & g_{2} \\
y & b_{2} & g_{3} \\
y & b_{2} & g_{4} \\
z & b_{3} & g_{5} \\
z & \underbrace{b_{3}}_{\text {invite }} & g_{6}
\end{array}\right] \stackrel{\text { output }}{\longrightarrow}
$$

For any given input matrix, there will be as many output matrices as there are ways of pairing every boy with two girls that he invited. So if we identify the "alternatives" of a sentence with its potential outputs, as I have here following insights in Charlow 2014, then $\delta_{i}$ delivers exactly the same set of pair-list alternatives that the iterative universal of (55) does! And as indicated in Section 5.3, this already takes us a long way toward an analysis of pair-list questions and embedded clauses. The missing ingredients are just a theory of questions and a theory of scope that allows alternatives to percolate over island boundaries (à la Kratzer \& Shimoyama 2002).

It is in some sense unsurprising that $\delta_{i}$ should deliver "pair-list" outputs despite being non-iterative, given that plural dynamic frameworks are motivated in large part by the need to keep track of quantificational dependencies, as in (9oa, b). On the relevant readings, the second clauses are interpreted in the distributive contexts of the first clauses. That is, (90a) is true if each student who wrote an article sent it (the article he wrote) to L\&P; and (9ob) is true if they each thought that writing it would be easier than studying. As in (89), the row-wise relations between discourse referents will enforce a writing relationship between the students stored in column 1 and the papers stored in column 2. Then when each column 1 individual is retrieved by the subsequent plural pronoun, and the assignment matrix restricted to his particular row, the singular pronoun directed toward column 2 will refer to the paper he wrote.

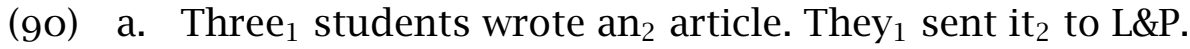

[Krifka 1996, (1)]

b. Most $_{1}$ students wrote $\mathrm{a}_{2}$ paper. They ${ }_{1}$ thought that writing it ${ }_{2}$ would be easier than studying for the exam. [Asher \& Wang 2003, (5b)] 
The problem is that clauses like (89) should not license pair-list phenomena, given the infelicity of pair-list answers to questions like (91) and the absence of Schlenker truth conditions for conditionals like (92). Nor should they be licensed by analogous clauses involving most or any quantifiers other than lexical, distributive universals. This is the case even though such clauses generate quantificational dependencies isomorphic to pair-lists that persist across sentence boundaries, as shown by the examples in (90). Thus, whatever is responsible for the availability of pair-list readings of questions and embedded clauses, it cannot be as general as the mechanisms responsible for dependent anaphora.

(91) Which two girls did the three boys invite?

a. They invited Ann and Bee.

b. They each invited Ann and Bee.

c. \#Alex invited Ann and Ada; Bill, Bee and Bev; and Carl, Cat and Cam. [cf. (21)]

(92) If the three boys invited two girls, the party will be a success.

a. $\quad\left(\forall x \ll \iota_{3}\right.$-boys. $\exists y$ : two-girls . invite $\left.y x\right) \Rightarrow$ success $\mathrm{p}$

b. $\# \exists f: \iota_{3}$-boys $\rightarrow$ two-girls . $\left(\forall x \ll \iota_{3}\right.$-boys . invite $\left.f(x) x\right) \Rightarrow$ success $\mathrm{p}$ [cf. (33)]

I should hasten to point out that to my knowledge, neither Brasoveanu nor any other plural dynamic semanticist has ever claimed that the sorts of dependency structures at issue in (90) are the same sorts of dependency structures at issue in (91) and (92), though it seems a perfectly reasonable hypothesis. The point is just that any DP that can be interpreted distributively appears to be capable of establishing dependencies that are available for subsequent subordination. So if we were inclined to derive pair-list readings via the same operation that builds distributive dependencies elsewhere (i.e., something like $\delta_{i}$ ), we'd be left without an explanation for the tight relationship between pair-lists and universals. ${ }^{17}$

In addition, $\delta_{i}$ still evaluates each individual of its restrictor independently. As a result, it does not leave any room for internal adjectives to

17 I should also add that the fragment developed here does not immediately extend to sentences like the ones in (9o). Reconciling the approach to pair-list dependencies here with successful approaches to dependency anaphora is a topic for future research. 
Incremental quantification

compare discourse referents assigned to distinct elements of the domain. This brings us to Brasoveanu's second innovation: lexicalized universals distribute predicates over pairs of the individuals denoted by their restrictors. By itself, this approach to internal readings runs straight through one track of the literature on same and different (e.g., Carlson 1987, Moltmann 1992, Beck 2000), but PCDRT offers novel formal possibilities for driving the two variables of the universal quantifier through the composition of the sentence in synchronized animation.

For example, the sentence in (93a) will spool up for every pair of distinct boys (say, John and Fred) a pair of dynamic updates, as schematically represented in (93b).

(93) a. Every boy recited a different poem.

[Brasoveanu 2011, (3)]

b. $\begin{aligned}\langle\lambda G H . G[x] H \wedge \text { poem } x \wedge \text { recite } x \mathrm{j}, \\ \lambda G H . G[y] H \wedge \text { poem } y \wedge \text { recite } y \mathrm{f}\rangle\end{aligned}$

In the scope of the universal, everything will feed into the two updates completely simultaneously and independently, much as in Alternative Semantics, unless the sentence contains an appropriately tuned internal adjective, like different. When different enters into the semantic composition, it sequences these two parallel updates made available by the universal, and performs its usual anaphoric test on the values of the variables chosen to witness John's and Fred's poems. If for every pair of updates (i.e., every pair of boys) it is possible to find distinct poems $x$ and $y$ that the two boys read respectively, then the entire sentence will be true.

So for Brasoveanu, different provides the grammar's only bridge between what would otherwise constitute redundant and independent updates set in motion by a universal quantifier. That bridge, as he engineers it, is erected from the same dynamic architecture that licenses cross-sentential anaphora.

The current proposal also derives internal readings from the potential for external ones, but it does so without hypothesizing any special link between distributors and comparators. There is no need to supplement different with a mechanism for collapsing quantificational alternatives. The comparison between poems just is cross-sentential anaphora, made possible by whatever dynamic mechanism makes sense out of sequences like (87).

Less conceptually, this analysis extends immediately to cases of asymmetric comparison like (13-16), exemplified below by (94). If distributive 
quantification is quantification over propositions in sequence, then it is expected that some anaphoric items are sensitive to the order in which the quantification happens. Short of postulating a second meaning for the universal which quantifies in duplicate over ordered pairs of alternatives, it is not obvious how to explain the felicity of (94) in a Brasoveanuan setting.

(94) ${ }^{\gamma}$ Every generation lives in a more Orwellian world.

[repeated from (14c)]

That such asymmetric internal readings share the same distributional restrictions as symmetric internal readings like (93) is a reason to think that the two interpretations are derived from the same compositional processes. And that internal readings in general arise in exactly the configurations that support pair-list answers to questions and pair-list witnesses for embedded clauses is evidence that one and the same mechanism is at work in every case. I have argued here that that mechanism is iterated dynamic conjunction.

\section{A Guided derivations}

\section{A.1 Monad laws}

It will be convenient to observe that $\eta$ and $\star$ respect the following identities, known to category theorists and functional programmers as the "monad laws":

i. $\eta x \star f=f x$

ii. $m \star \eta=m$

iii. $(m \star f) \star g=m \star(\lambda x . f x \star g)$

From these identities, it is straightforward to prove the following equivalences, which will simplify the coming derivations:

$$
\begin{aligned}
& \eta g / \eta x=\eta g \star\left(\lambda g^{\prime} . \eta x \star\left(\lambda x^{\prime} . \eta\left(g^{\prime} x^{\prime}\right)\right)\right) \\
& =\eta g \star\left(\lambda g^{\prime} . \eta\left(g^{\prime} x\right)\right) \\
& =\eta(g x) \\
& \eta x \backslash \eta g=\eta x \star\left(\lambda x^{\prime} . \eta g \star\left(\lambda g^{\prime} . \eta\left(g^{\prime} x^{\prime}\right)\right)\right) \\
& =\eta x \star\left(\lambda x^{\prime} \cdot \eta\left(g x^{\prime}\right)\right) \\
& =\eta(g x)
\end{aligned}
$$


Incremental quantification

$$
\begin{aligned}
\llbracket \phi \rrbracket ; \llbracket \psi \rrbracket & =\llbracket \phi \rrbracket \backslash(\eta(\lambda p q \cdot q \wedge p) / \llbracket \psi \rrbracket) \\
& =\llbracket \phi \rrbracket \backslash(\eta(\lambda p q \cdot q \wedge p) \star(\lambda h \cdot \llbracket \psi \rrbracket \star(\lambda p \cdot \eta(h p))) \\
& =\llbracket \phi \rrbracket \backslash(\llbracket \psi \rrbracket \star(\lambda p \cdot \eta(\lambda q \cdot q \wedge p))) \\
& =\llbracket \phi \rrbracket \star(\lambda q \cdot(\llbracket \psi \rrbracket \star(\lambda p \cdot \eta(\lambda q \cdot q \wedge p))) \star(\lambda h \cdot \eta(h q))) \\
& =\llbracket \phi \rrbracket \star(\lambda q \cdot \llbracket \psi \rrbracket \star(\lambda p \cdot \eta(\lambda q \cdot q \wedge p) \star(\lambda h \cdot \eta(h q)))) \\
& =\llbracket \phi \rrbracket \star(\lambda q \cdot \llbracket \psi \rrbracket \star(\lambda p \cdot \eta(q \wedge p))) \\
& =\llbracket \phi \rrbracket \star\left(\lambda q s^{\prime} \cdot \bigcup\left\{\left\{\left\langle q \wedge p, s^{\prime \prime}\right\rangle\right\} \mid\left\langle p, s^{\prime \prime}\right\rangle \in \llbracket \psi \rrbracket s^{\prime}\right\}\right) \\
& =\llbracket \phi \rrbracket \star\left(\lambda q s^{\prime} \cdot\left\{\left\langle q \wedge p, s^{\prime \prime}\right\rangle \mid\left\langle p, s^{\prime \prime}\right\rangle \in \llbracket \psi \rrbracket s^{\prime}\right\}\right) \\
& =\lambda s \cdot \bigcup\left\{\left\{\left\langle q \wedge p, s^{\prime \prime}\right\rangle \mid\left\langle p, s^{\prime \prime}\right\rangle \in \llbracket \psi \rrbracket s^{\prime}\right\} \mid\left\langle q, s^{\prime}\right\rangle \in \llbracket \phi \rrbracket s\right\} \\
& =\lambda s \cdot\left\{\left\langle q \wedge p, s^{\prime \prime}\right\rangle \mid\left\langle q, s^{\prime}\right\rangle \in \llbracket \phi \rrbracket s,\left\langle p, s^{\prime \prime}\right\rangle \in \llbracket \psi \rrbracket s^{\prime}\right\}
\end{aligned}
$$

\section{A.2 Example derivations}

\section{Mary kissed a boy.}

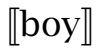

$$
\begin{aligned}
& =\eta \text { boy } \\
& =\lambda s \cdot \bigcup\left\{m s \mid m \in{ }^{\ulcorner} \eta \text { boy }^{\top}\right\} \\
& =\lambda s . \bigcup\left\{m s \mid m \in\left\{\eta x \mid \operatorname{TRUE}_{\varepsilon}(\eta \text { boy } / \eta x)\right\}\right\} \\
& =\lambda s . \bigcup\left\{m s \mid m \in\left\{\eta x \mid \operatorname{TRUE}_{\varepsilon}(\eta(\text { boy } x))\right\}\right\} \\
& =\lambda s . \bigcup\left\{m s \mid m \in\left\{\eta x \mid \exists s^{\prime} .\left\langle\mathrm{T}, s^{\prime}\right\rangle \in\{\langle\text { boy } x, \varepsilon\rangle\}\right\}\right\} \\
& =\lambda s . \bigcup\{m s \mid m \in\{\eta x \mid \text { boy } x\}\} \\
& =\lambda s \cdot \bigcup\{\{\langle x, s\rangle\} \mid \text { boy } x\} \\
& =\lambda s \cdot\{\langle x, s\rangle \mid \operatorname{boy} x\}
\end{aligned}
$$

$\llbracket[\text { a boy }]^{\triangleright} \rrbracket$ 


$$
\begin{aligned}
& =(\lambda s \cdot\{\langle x, s\rangle \mid \text { boy } x\}) \star(\lambda x s \cdot \eta x s \cdot x) \\
& =\lambda s \cdot \bigcup\left\{\left\{\left\langle x, s^{\prime} \cdot x\right\rangle\right\} \mid\left\langle x, s^{\prime}\right\rangle \in\{\langle x, s\rangle \mid \text { boy } x\}\right\} \\
& =\lambda s \cdot\{\langle x, s \cdot x\rangle \mid \text { boy } x\}
\end{aligned}
$$

【kissed】

$=\eta$ kiss

$\llbracket\left[\right.$ kissed / $\left.[\text { a boy }]^{\triangleright}\right] \rrbracket$

$$
\begin{aligned}
& =\eta \text { kiss } / \lambda s \cdot\{\langle x, s \cdot x\rangle \mid \text { boy } x\} \\
& =\eta \text { kiss } \star(\lambda g \cdot(\lambda s \cdot\{\langle x, s \cdot x\rangle \mid \text { boy } x\}) \star(\lambda x \cdot \eta(g x))) \\
& =\eta \text { kiss } \star\left(\lambda g s \cdot \bigcup\left\{\left\{\left\langle g x, s^{\prime}\right\rangle\right\} \mid\left\langle x, s^{\prime}\right\rangle \in\{\langle x, s \cdot x\rangle \mid \text { boy } x\}\right\}\right) \\
& =\eta \text { kiss } \star(\lambda g s \cdot\{\langle g x, s \cdot x\rangle \mid \text { boy } x\}) \\
& =\lambda s .\{\langle\operatorname{kiss} x, s \cdot x\rangle \mid \text { boy } x\}
\end{aligned}
$$

$\llbracket\left[\right.$ Mary $\backslash\left[\right.$ kissed $\left.\left./[\text { a boy }]^{\triangleright}\right]\right] \rrbracket$

$$
\begin{aligned}
& =\eta \mathrm{m} / \lambda s \cdot\{\langle\operatorname{kiss} x, s \cdot x\rangle \mid \text { boy } x\} \\
& =\eta \mathrm{m} \star(\lambda y \cdot(\lambda s \cdot\{\langle\operatorname{kiss} x, s \cdot x\rangle \mid \text { boy } x\}) \star(\lambda h \cdot \eta(h y))) \\
& =\eta \mathrm{m} \star\left(\lambda y s \cdot \bigcup\left\{\left\{\left\langle h y, s^{\prime}\right\rangle\right\} \mid\left\langle h, s^{\prime}\right\rangle \in\{\langle\operatorname{kiss} x, s \cdot x\rangle \mid \text { boy } x\}\right\}\right) \\
& =\eta \mathrm{m} \star(\lambda y s \cdot\{\langle\operatorname{kiss} x y, s \cdot x\rangle \mid \text { boy } x\}) \\
& =\lambda s .\{\langle\operatorname{kiss} x \mathrm{~m}, s \cdot x\rangle \mid \text { boy } x\}
\end{aligned}
$$

\section{Every girl kissed a boy}

【girl】

$$
=\eta \operatorname{girl}
$$

【[every girl] 』 
Incremental quantification

$$
\begin{aligned}
& =\lambda k \cdot ;\left\{m \star k \mid m \in{ }^{\prime} \eta \operatorname{girl}\right\} \\
& =\lambda k \cdot ;\left\{m \star k \mid m \in\left\{\eta y \mid \operatorname{TRUE}_{\varepsilon}(\eta \operatorname{girl} / \eta y)\right\}\right\} \\
& =\lambda k \cdot ;\left\{m \star k \mid m \in\left\{\eta y \mid \mathrm{TRUE}_{\varepsilon}(\eta(\operatorname{girl} y))\right\}\right\} \\
& =\lambda k \cdot ;\left\{m \star k \mid m \in\left\{\eta y \mid \exists s^{\prime} .\left\langle\mathrm{T}, s^{\prime}\right\rangle \in\{\langle\operatorname{girl} y, \varepsilon\rangle\}\right\}\right\} \\
& =\lambda k \cdot ;\{m \star k \mid m \in\{\eta y \mid \operatorname{girl} y\}\} \\
& =\lambda k \cdot ;\{\eta y \star k \mid \operatorname{girl} y\} \\
& =\lambda k \cdot ;\{k y \mid \operatorname{girl} y\}
\end{aligned}
$$

$\llbracket[\text { every girl }]^{\vee} \rrbracket$

$$
\begin{aligned}
& =\lambda k .\left(\lambda k^{\prime} . ;\left\{k^{\prime} y \mid \operatorname{girl} y\right\}\right)(\lambda x s . k x s \cdot x) \\
& =\lambda k . ;\{\lambda s . k y s \cdot y \mid \operatorname{girl} y\}
\end{aligned}
$$

$\llbracket\left[t_{1} \backslash\left[\right.\right.$ kissed $\left.\left./[\text { a boy }]^{\triangleright}\right]\right] \rrbracket$

$$
=\lambda s .\{\langle\operatorname{kiss} x(g 1), s \cdot x\rangle \mid \text { boy } x\}
$$

$\llbracket[\text { every girl }]_{1}^{\triangleright}\left[t_{1} \backslash\left[\right.\right.$ kissed $\left.\left./[\text { a boy }]^{\triangleright}\right]\right] \rrbracket$

$$
\begin{aligned}
& =(\lambda k . ;\{\lambda s \cdot k y s \cdot y \mid \operatorname{girl} y\})\left(\lambda y \cdot \llbracket\left[t_{1} \backslash\left[\operatorname{kissed} /\left[\mathrm{a} \text { boy }{ }^{\triangleright}\right]\right] \rrbracket^{g[1 \mapsto y]}\right)\right. \\
& =(\lambda k . ;\{\lambda s . k y s \cdot y \mid \operatorname{girl} y\})(\lambda y s .\{\langle\operatorname{kiss} x y, s \cdot x\rangle \mid \text { boy } x\}) \\
& =;\{\lambda s .\{\langle\operatorname{kiss} x y, s \cdot y \cdot x\rangle \mid \operatorname{boy} x\} \mid \operatorname{girl} y\} \\
& =\lambda s \cdot\left\{\left\langle\operatorname{kissg}_{1} x, s \cdot \mathrm{g}_{1} \cdot x\right\rangle \mid \text { boy } x\right\} \text {; } \\
& \lambda s .\left\{\left\langle\operatorname{kissg}_{2} x, s \cdot \mathrm{g}_{2} \cdot x\right\rangle \mid \text { boy } x\right\} \text {; } \\
& \lambda s \cdot\left\{\left\langle\operatorname{kiss}_{3} x, s \cdot \mathrm{g}_{3} \cdot x\right\rangle \mid \text { boy } x\right\} \\
& =\lambda s \cdot\left\{\left\langle\operatorname{kissg}_{1} x \wedge \operatorname{kissg}_{2} y \wedge \operatorname{kissg}_{3} z, s \cdot \mathrm{g}_{1} \cdot x \cdot \mathrm{g}_{2} \cdot y \cdot \mathrm{g}_{3} \cdot z\right\rangle \mid x, y, z \in \text { boy }\right\}
\end{aligned}
$$

\section{Every girl kissed a different boy}

$\llbracket[$ different / boy $] \rrbracket$ 
$=\llbracket$ different $\rrbracket \star(\lambda g . \eta$ boy $\star(\lambda h \cdot \eta(g h)))$

$=\llbracket$ different $\rrbracket \star(\lambda g \cdot \eta(g$ boy $))$

$=(\lambda s \cdot \eta(\lambda P x \cdot P x \wedge x \notin s) s) \star(\lambda g \cdot \eta(g$ boy $))$

$=\lambda s .\{\langle\lambda x$. boy $x \wedge x \notin s, s\rangle\}$

$\llbracket[\text { a }[\text { different / boy }]]^{\triangleright} \rrbracket$

$=\lambda s \cdot\{\langle x, s \cdot x\rangle \mid$ boy $x, \mathrm{x} \notin \mathrm{s}\}$

$\left.\left.\llbracket\left[t_{1} \backslash[\text { kissed / [a [different / boy }]\right]^{\triangleright}\right]\right] \rrbracket$

$=\lambda s \cdot\{\langle\operatorname{kiss} x(g 1), s \cdot x\rangle \mid$ boy $x, x \notin s\}$

$\left.\left.\llbracket[\text { every girl }]_{1}^{\triangleright}\left[t_{1} \backslash[\text { kissed / [a [different boy }]\right]^{\triangleright}\right]\right] \rrbracket$

$=;\{\lambda s .\{\langle\operatorname{kiss} x y, s \cdot y \cdot x\rangle \mid$ boy $x, x \notin s\} \mid \operatorname{girl} y\}$

$=\lambda s \cdot\left\{\left\langle\operatorname{kiss}_{1} x, s \cdot \mathrm{g}_{1} \cdot x\right\rangle \mid\right.$ boy $\left.x, x \notin s\right\}$;

$\lambda s \cdot\left\{\left\langle\operatorname{kiss}_{2} x, s \cdot \mathrm{g}_{2} \cdot x\right\rangle \mid\right.$ boy $\left.x, x \notin s\right\}$;

$\lambda s .\left\{\left\langle\operatorname{kiss}_{3} x, s \cdot \mathrm{g}_{3} \cdot x\right\rangle \mid\right.$ boy $\left.x, x \notin s\right\}$

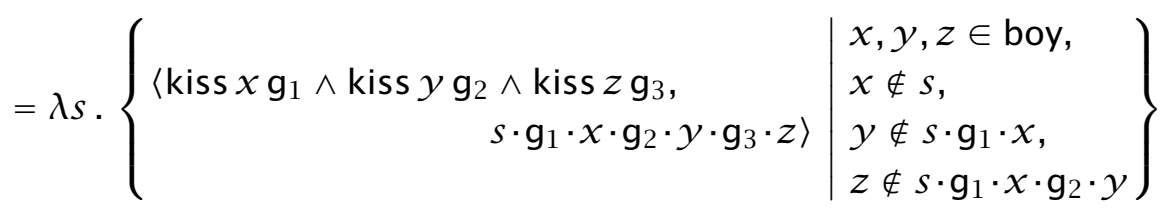

If every girl kisses a (certain) boy, the party will be a success.

【[if $\left.t_{1}\right] \rrbracket$

$=\left(\lambda m n s .\left\{\left\langle\forall\left\langle q, s^{\prime}\right\rangle \in m s . q \Rightarrow \operatorname{TRUE}_{s^{\prime}} n, s\right\rangle\right\}\right)(\lambda s .\{\langle g 1, s\rangle\})$

$=\lambda n s .\left\{\left\langle\forall\left\langle q, s^{\prime}\right\rangle \in\{\langle g 1, s\rangle\} \cdot q \Rightarrow \operatorname{TRUE}_{s} n, s\right\rangle\right\}$

$=\lambda n s .\left\{\left\langle g 1 \Rightarrow \operatorname{TRUE}_{s} n, s\right\rangle\right\}$

$\llbracket[$ the party will be a success] $]$

$=\lambda s .\{\langle$ success $p, s\rangle\}$

$\llbracket\left[\left[\right.\right.$ if $\left.t_{1}\right][\ldots$ success $\left.]\right] \rrbracket$ 
Incremental quantification

$$
\begin{aligned}
& =\left(\lambda n s .\left\{\left\langle g 1 \Rightarrow \operatorname{TRUE}_{s} n, s\right\rangle\right\}\right)(\lambda s .\{\langle\text { success } p, s\rangle\}) \\
& =\lambda s \cdot\left\{\left\langle g 1 \Rightarrow \operatorname{TRUE}_{s}(\lambda s .\{\langle\text { success } p, s\rangle\}), s\right\rangle\right\} \\
& =\lambda s .\{\langle g 1 \Rightarrow \text { success } p, s\rangle\}
\end{aligned}
$$

$\llbracket[\text { every girl kisses a boy }]^{\dagger} \rrbracket$

$$
\begin{aligned}
& =\lambda k \cdot\left(\lambda s \cdot\left\{\begin{array}{c}
\left\langle\operatorname{kiss~g}_{1} x \wedge \operatorname{kissg}_{2} y \wedge \operatorname{kissg}_{3} z,\right. \\
\left.s \cdot \mathrm{g}_{1} \cdot x \cdot \mathrm{g}_{2} \cdot y \cdot \mathrm{g}_{3} \cdot z\right\rangle
\end{array} \mid x, y, z \in \text { boy }\right\}\right) \star k
\end{aligned}
$$

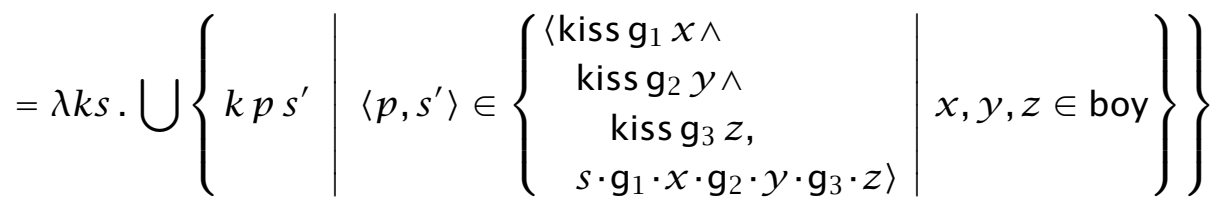

$$
\begin{aligned}
& =\lambda k s \cdot \bigcup\left\{\begin{array}{c|c}
k\left(\operatorname{kissg}_{1} x \wedge \operatorname{kissg}_{2} y \wedge \operatorname{kissg}_{3} z\right) & x, y, z \in \text { boy } \\
\left(s \cdot \mathrm{g}_{1} \cdot x \cdot \mathrm{g}_{2} \cdot y \cdot \mathrm{g}_{3} \cdot z\right) & x,
\end{array}\right.
\end{aligned}
$$

$\llbracket\left[[\text { every girl kisses a boy }]_{1}^{\dagger}\left[\left[\right.\right.\right.$ if $\left.t_{1}\right][$... success $\left.\left.]\right]\right] \rrbracket$

$$
\begin{aligned}
& =\left(\llbracket[\text { every girl kisses a boy }]^{\dagger} \rrbracket\right)\left(\lambda q \cdot \llbracket\left[\left[\text { if } t_{1}\right][\ldots \text { success }]\right] \rrbracket^{g[1 \mapsto q]}\right) \\
& =\left(\llbracket[\text { every girl kisses a boy }]^{\dagger} \rrbracket\right)(\lambda q s .\{\langle q \Rightarrow \text { success } p, s\rangle\}) \\
& =\lambda s \cdot \bigcup\left\{\left\{\begin{array}{c}
\left\langle\text { kiss g }_{1} x \wedge \text { kiss g }_{2} y \wedge{\text { kiss } g_{3} z \Rightarrow \text { success } \mathrm{p},}_{\left.s \cdot \mathrm{g}_{1} \cdot x \cdot \mathrm{g}_{2} \cdot y \cdot \mathrm{g}_{3} \cdot z\right\rangle}\right.
\end{array}\right\} \mid x, y, z \in \text { boy }\right\}
\end{aligned}
$$

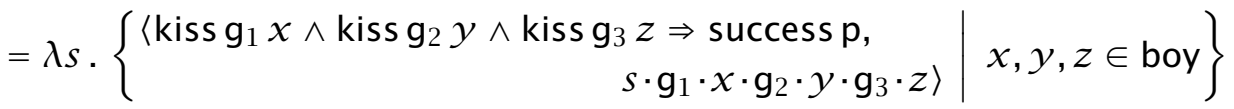

\section{References}

Alonso-Ovalle, Luis. 2006. Disjunction in alternative semantics. Amherst, MA: University of Massachusetts Amherst PhD Dissertation.

Alonso-Ovalle, Luis. 2009. Counterfactuals, correlatives, and disjunction. Linguistics and Philosophy 32(2). 207-244. http://dx.doi.org/10.1007/ s10988-009-9059-0.

Asher, Nicholas \& Linton Wang. 2003. Ambiguity and anaphora with plurals in discourse. Semantics and Linguistic Theory (SALT) 13. 19-36. 
Barker, Chris. 2002. Continuations and the nature of quantification. Natural Language Semantics 10(3). 211-242. http://dx.doi.org/10.1023/A: 1022183511876.

Barker, Chris. 2007. Parasitic scope. Linguistics and Philosophy 30(4). 407444. http://dx.doi.org/10.1007/s10988-007-9021-y.

Barker, Chris. 2009. Reconstruction as delayed evaluation. In Erhard Hinrichs \& John Nerbonne (eds.), Theory and evidence in semantics, chap. 1, 1-28. Stanford, CA: CSLI Publications.

Beck, Sigrid. 200o. The semantics of different: Comparison operator and relational adjective. Linguistics and Philosophy 23(2). 101-139. http://dx. doi.org/10.1023/A:1005566722022.

Beghelli, Filippo. 1997. The syntax of distributivity and pair-list readings. In Anna Szabolcsi (ed.), Ways of scope taking, vol. 65 (Studies in Linguistics and Philosophy), chap. 10, 349-408. Dordrecht: Kluwer Academic.

Beghelli, Filippo \& Tim Stowell. 1997. Distributivity and negation: The syntax of each and every. In Anna Szabolcsi (ed.), Ways of scope taking, vol. 65 (Studies in Linguistics and Philosophy), 71-107. Dordrecht: Kluwer Academic.

Belnap, Jr., Nuel D. 1982. Questions and answers in Montague Grammar. In Stanley Peters \& Esa Saarinen (eds.), Processes, beliefs, and questions, vol. 16 (Synthese Language Library), 165-198. Springer Netherlands. http: //dx.doi.org/10.1007/978-94-015-7668-0_6.

van den Berg, Martin. 1996a. Dynamic generalized quantifiers. In Jaap van der Does \& Jan van Eijck (eds.), Quantifiers, logic, and language, vol. 54 (Lecture Notes), chap. 3, 63-94. Stanford, CA: CSLI Publications.

van den Berg, Martin. 1996b. Some aspects of the internal structure of discourse: the dynamics of nominal anaphora. Amsterdam: University of Amsterdam $\mathrm{PhD}$ thesis.

Brasoveanu, Adrian. 2007. Structured nominal and modal reference. New Brunswick, NJ: Rutgers University PhD thesis.

Brasoveanu, Adrian. 2008. Donkey pluralities: Plural information states versus non-atomic individuals. Linguistics and Philosophy 31(2). 129-209. http: //dx.doi.org/10.1007/s10988-008-9035-0.

Brasoveanu, Adrian. 2011. Sentence-internal different as quantifier-internal anaphora. Linguistics and Philosophy 34(2). 93-168. http://dx.doi.org/10. 1007/s10988-011-9096-3.

Brasoveanu, Adrian. 2012. Modified numerals as post-suppositions. Journal of Semantics 30(2). 155-209. http://dx.doi.org/10.1093/jos/ffsoo3. 
Incremental quantification

Brasoveanu, Adrian \& Jakub Dotlačil. 2012. Licensing sentence-internal readings in English: An experimental study. Amsterdam Colloquium 18. 122132. http://dx.doi.org/10.1007/978-3-642-31482-7_13.

Brasoveanu, Adrian \& Donka Farkas. 2011. How indefinites choose their scope. Linguistics and Philosophy 34(1). 1-55. http://dx.doi.org/10.1007/s10988o11-9092-7.

Bumford, Dylan. 2013. Incremental quantification. Unpublished manuscript, New York University.

Bumford, Dylan \& Chris Barker. 2013. Association with distributivity and the problem of multiple antecedents for singular different. Linguistics and Philosophy 36(5). 355-369. http://dx.doi.org/10.1007/s10988-013-9139-Z.

Carlson, Greg. 1987. Same and different: Some consequences for syntax and semantics. Linguistics and Philosophy 10(4). 531-565. http://dx.doi.org/10. 1007/BFoo628069.

Charlow, Simon. 2014. On the semantics of exceptional scope. New York, NY: New York University PhD Dissertation.

Chierchia, Gennaro. 1992. Functional wh and weak crossover. West Coast Conference on Formal Linguistics (WCCFL) 10. Dawn Bates (ed.). 75-90.

Chierchia, Gennaro. 1993. Questions with quantifiers. Natural Language Semantics 1(2). 181-234. http://dx.doi.org/10.1007/BFoo372562.

Chierchia, Gennaro. 2001. A puzzle about indefinites. In Carlo Cecchetto, Gennaro Chierchia \& Maria Teresa Guasti (eds.), Semantic interfaces (Lecture Notes), 51-89. Stanford, CA: CSLI Publications.

Ciardelli, Ivano. 2009. Inquisitive semantics and intermediate logics. Amsterdam: University of Amsterdam MSc Thesis.

Ciardelli, Ivano, Jeroen Groenendijk \& Floris Roelofsen. 2013. Inquisitive semantics: A new notion of meaning. Language and Linguistics Compass 7(9). 459-476. http://dx.doi.org/10.1111/lnc3.12037.

Ciardelli, Ivano \& Floris Roelofsen. 2011. Inquisitive logic. Journal of Philosophical Logic 40(1). 55-94. http://dx.doi.org/10.1007/s10992-010-9142-6.

Dayal, Veneeta. 1996. Locality in WH quantification: Questions and relative clauses in Hindi. Dordrecht: Kluwer Academic.

Dekker, Paul. 1994. Predicate logic with anaphora. Semantics and Linguistic Theory (SALT) 4. 79-95.

Dotlačil, Jakub. 2010. Anaphora and distributivity: A study of same, different, reciprocals and others. Utrecht: Utrecht University $\mathrm{PhD}$ thesis. 
Dowty, David. 1985. A unified indexical analysis of same and different: A response to Stump and Carlson. Paper presented at the University of Texas Workshop on Syntax and Semantics. Austin, TX.

Eijck, Jan van. 2001. Incremental dynamics. Journal of Logic, Language and Information 10(3). 319-351. http://dx.doi.org/10.1023/A:1011251627260.

Engdahl, Elisabet. 1980. The syntax and semantics of questions in Swedish. Amherst, MA: University of Massachusetts Amherst PhD Dissertation.

Engdahl, Elisabet. 1985. Interpreting questions. In David Dowty, Lauri Karttunen \& Arnold Zwicky (eds.), Natural language parsing: Psychological, computational, and theoretical perspectives (Studies in Natural Language Processing), chap. 2, 67-93. Cambridge, UK: Cambridge University Press. http://dx.doi.org/10.1017/CBO9780511597855.003.

Engdahl, Elisabet. 1986. Constituent questions: The syntax and semantics of questions with special reference to Swedish. Dordrecht: D. Reidel Publishing Company.

Farkas, Donka. 1981. Quantifier scope and syntactic islands. Chicago Linguistics Society (CLS) 17. 59-66.

Fine, Kit. 2012. Counterfactuals without possible worlds. The Journal of Philosophy 109(3). 221-246.

Fine, Kit. 2013. Truth-maker semantics for intuitionistic logic. Journal of Philosophical Logic. 1-29. http://dx.doi.org/10.1007/s10992-013-9281-7.

Gawron, Jean Mark \& Andrew Kehler. 2003. Respective answers to coordinated questions. Semantics and Linguistic Theory (SALT) 13. 91-108.

Geach, Peter. 1964. Referring expressions again. Analysis 24(5). 172-175. http: //dx.doi.org/10.1093/analys/24.5.172.

Geurts, Bart. 2000. Indefinites and choice functions. Linguistic Inquiry 31(4). 731-738. http://dx.doi.org/10.1162/o02438900554550.

Gil, David. 1982. Quantifier scope, linguistic variation, and natural language semantics. Linguistics and Philosophy 5(4). 421-472. http://dx.doi.org/10. 1007/BFo0355582.

Groenendijk, Jeroen \& Floris Roelofsen. 2009. Inquisitive semantics and pragmatics. Paper presented at the Workshop on Language, Communication, and Rational Agency. Stanford, CA: Stanford University.

Groenendijk, Jeroen \& Martin Stokhof. 1984. Studies on the semantics of questions and the pragmatics of answers. Amsterdam: University of Amsterdam $\mathrm{PhD}$ thesis.

Groenendijk, Jeroen \& Martin Stokhof. 1989. Type-shifting rules and the semantics of interrogatives. In Gennaro Chierchia, Barbara Partee \& Ray- 
Incremental quantification

mond Turner (eds.), Properties, types and meaning: Semantic issues (Studies in Linguistics and Philosophy 39), 21-69. Dordrecht: Kluwer Academic. Groenendijk, Jeroen \& Martin Stokhof. 1990. Dynamic Montague Grammar. In László Kálmán \& László Pólos (eds.), Papers from the second symposium on logic and language, 3-48. Budapest: Akadémiai Kiadó.

Groenendijk, Jeroen \& Martin Stokhof. 1991. Dynamic predicate logic. Linguistics and Philosophy 14. 39-100. http://dx.doi.org/10.1007/BFoo628304.

de Groote, Phillippe. 2006. Towards a Montagovian account of dynamics. Semantics and Linguistic Theory (SALT) 16. 1-16.

Hamblin, C. L. 1958. Questions. Australasian Journal of Philosophy 36. 159168. http://dx.doi.org/10.1080/00048405885200211.

Hamblin, C. L. 1973. Questions in Montague English. Foundations of Language 10(1). 41-53. http://dx.doi.org/10.1016/b978-0-12-545850-4.50014-5.

Heim, Irene. 1985. Notes on comparatives and related matters. Unpublished manuscript, University of Texas at Austin. http://semanticsarchive.net/ Archive/zcoZjYoM.

Heim, Irene, Howard Lasnik \& Robert May. 1991. Reciprocity and plurality. Linguistic Inquiry 22(1). 63-102. http://www.jstor.org/stable/4178708.

Hintikka, Jaakko. 1986. The semantics of a certain. Linguistic Inquiry 17(2). 331-336. http://www.jstor.org/stable/4178489.

Jacobson, Pauline. 1994. Binding connectivity in copular sentences. Semantics and Linguistic Theory (SALT) 4. 161-178.

Jacobson, Pauline. 1999. Towards a variable-free semantics. Linguistics and Philosophy 22(2). 117-184. http://dx.doi.org/10.1023/A:1005464228727.

Karttunen, Lauri. 1977. Syntax and semantics of questions. Linguistics and Philosophy 1(1). 3-44. http://dx.doi.org/10.1007/BFo0351935.

Keenan, Edward. 1987. Unreducible $n$-ary quantifiers in natural language. In Peter Gärdenfors (ed.), Generalized quantifiers, vol. 31 (Studies in Linguistics and Philosophy), 109-150. Dordrecht: D. Reidel Publishing Company. http://dx.doi.org/10.1007/978-94-009-3381-1_5.

Kratzer, Angelika. 1998. Scope or pseudoscope? Are there wide-scope indefinites? In Susan Rothstein (ed.), Events and grammar, 163-196. Dordrecht: Kluwer.

Kratzer, Angelika \& Junko Shimoyama. 2002. Indeterminate pronouns: The view from Japanese. Tokyo Conference on Psycholinguistics 3. Yukio Otsu (ed.). 1-25.

Krifka, Manfred. 1992. Definite NPs aren't quantifiers. Linguistic Inquiry 23(1). 156-163. http://www.jstor.org/stable/4178762. 
Krifka, Manfred. 1996. Parametrized sum individuals for plural anaphora. Linguistics and Philosophy 19(6). 555-598. http://dx.doi.org/10.1007/ BFoo632708.

Krifka, Manfred. 2001. Quantifying into question acts. Natural Language Semantics 9(1). 1-40. http://dx.doi.org/10.1023/A:1017903702063.

Link, Godehard. 1987. Generalized quantifiers and plurals. In Peter Gärdenfors (ed.), Generalized quantifiers, vol. 31 (Studies in Linguistics and Philosophy), 151-180. Dordrecht: D. Reidel Publishing Company. http: //dx.doi.org/10.1007/978-94-0o9-3381-1_6.

Mascarenhas, Salvador. 2009. Inquisitive semantics and logic. Amsterdam: University of Amsterdam MSc Thesis.

May, Robert. 1985. Logical form: Its structure and derivation. Cambridge, MA: MIT Press.

Moltmann, Friederike. 1992. Reciprocals and same/different: Towards a semantic analysis. Linguistics and Philosophy 15(4). 411-462. http://dx.doi. org/10.1007/BFoo627683.

Muskens, Reinhard. 1996. Combining Montague semantics and Discourse Representation. Linguistics and Philosophy 19(2). 143-186. http://dx.doi. org/10.1007/BFoo635836.

Nouwen, Rick. 2003. Plural pronominal anaphora in context: Dynamic aspects of quantification. Utrecht: Utrecht Institute for Linguistics OTS PhD thesis. Nouwen, Rick. 2007. On dependent pronouns and dynamic semantics. Journal of Philosophical Logic 36(2). 123-154. http://dx.doi.org/10.1007/s10992oo6-9029-8.

Partee, Barbara. 1986. Noun phrase interpretation and type-shifting principles. In Jeroen Groenendijk, Dick de Jongh \& Martin Stokhof (eds.), Studies in Discourse Representation Theory and the theory of generalized quantifiers, 115-144. Dordrecht: Foris.

Roberts, Craige. 1987. Modal subordination, anaphora, and distributivity. Amherst, MA: University of Massachusetts Amherst PhD Dissertation.

Roelofsen, Floris. 2013. Algebraic foundations for the semantic treatment of inquisitive content. Synthese 190(1). 79-102. http://dx.doi.org/10.1007/ S11229-013-0282-4.

Rooth, Mats \& Barbara Partee. 1982. Conjunction, type ambiguity and wide scope or. West Coast Conference on Formal Linguistics (WCCFL) 1. 353-362. Schlenker, Philippe. 1998. Skolem functions and the scope of indefinites. North East Linguistic Society (NELS) 28. Pius Tamanji \& Kiyomi Kusumoto (eds.). 
Incremental quantification

Schlenker, Philippe. 2006. Scopal independence: A note on branching and island-escaping readings of indefinites and disjunctions. Journal of Semantics 23(3). 281-314. http://dx.doi.org/10.1093/jos/ffloo5.

Schwarz, Bernhard. 2001. Two kinds of long-distance indefinites. Unpublished manuscript, University of Texas at Austin.

Schwarzschild, Roger. 1996. Pluralities. Vol. 61 (Studies in Linguistics and Philosophy). Dordrecht: Kluwer Academic.

Shan, Chung-chieh. 2001. Monads for natural language semantics. Student session, European Summer School in Logic, Language and Information (ESSLLI) 13. 285-298.

Sharvit, Yael. 1996. Functional dependencies and indirect binding. Semantics and Linguistic Theory (SALT) 6. 227-244.

Sharvit, Yael. 1997. The syntax and semantics of functional relative clauses. New Brunswick, NJ: Rutgers University PhD Dissertation.

Sharvit, Yael. 1999. Functional relative clauses. Linguistics and Philosophy 22(5). 447-478. http://dx.doi.org/10.1023/A:1005411720444.

Slade, Benjamin. 2011. Formal and philological inquiries into the nature of interrogatives, indefinites, disjunction, and focus in Sinhala and other languages. Champaign, IL: University of Illinois at Urbana-Champaign Ph.D. Dissertation.

Solomon, Mike. 2011. True distributivity and the functional interpretation of indefinites. Unpublished manuscript, New York University. http:// semanticsarchive.net/Archive/zkxN2M4M.

Spector, Benjamin. 2004. Distributivity and specific indefinites. Conference of the Student Organization of Linguistics in Europe (ConSOLE) XII. 155-170.

Srivastav, Veneeta. 1992. Two types of universal terms in questions. North East Linguistic Society (NELS) 22. 443-457.

von Stechow, Arnim. 1990. Layered traces. Paper presented at the Conference on Logic and Language, Révfülöp, Hungary.

Stump, Gregory. 1982. A GPSG fragment for “dependent nominals”. Unpublished manuscript, The Ohio State University.

Szabolcsi, Anna. 1997. Quantifiers in pair-list readings. In Anna Szabolcsi (ed.), Ways of scope taking, vol. 65 (Studies in Linguistics and Philosophy), chap. 9, 311-347. Dordrecht: Kluwer Academic.

Szabolcsi, Anna. 2010. Quantification (Research Surveys in Linguistics). Cambridge, UK: Cambridge University Press. 
Vermeulen, Cees F. M. 1993. Sequence semantics for dynamic predicate logic. Journal of Logic, Language and Information 2(3). 217-254. http://dx.doi. org/10.1007/BFo1050788.

Vermeulen, Cees F. M. 1995. Merging without mystery or: Variables in dynamics semantics. Journal of Philosophical Logic 24(4). 405-450. http: //dx.doi.org/10.1007/BFo1048354.

Wadler, Philip. 1994. Monads and composable continuations. Lisp and Symbolic Computation 7(1). 39-55. http://dx.doi.org/10.1007/BFo1019944.

Winter, Yoad. 2001. Flexibility principles in boolean semantics. Vol. 37 (Current Studies in Linguistics). Cambridge, MA: MIT Press.

Winter, Yoad. 2004. Functional quantification. Research on Language \& Computation 2(3). 331-363. http://dx.doi.org/10.1007/s11168-004-0909-1.

Zeevat, Henk. 1989. A compositional approach to Discourse Representation Theory. Linguistics and Philosophy 12(1). 95-131. http://dx.doi.org/10. 1007/BFoo627399.

Dylan Bumford

Department of Linguistics

New York University

10 Washington Place

New York, NY 10003

dbumford@nyu.edu 